\title{
The Kentucky SimSmoke Tobacco Control Policy Model of Smokeless Tobacco and Cigarette Use
}

\author{
Luz María Sánchez-Romero ${ }^{\mathbb{D}}$, Zhe Yuan, Yameng Li, David T. Levy* ${ }^{\mathbb{D}}$
}

\begin{abstract}
Background: Smokeless tobacco (SLT) prevalence was decreasing in Kentucky before 2007, but has since increased. This study examines the impact of policies on cigarette and SLT use by applying the SimSmoke tobacco control policy simulation model.

Methods: Using data from the large-scale Tobacco Use Supplement of the Current Population Survey (TUS-CPS) and information on state-specific tobacco policies, Kentucky SimSmoke is updated and extended to incorporate exclusive SLT and dual cigarette and SLT use. The model is validated using survey data through 2017. The model was used to estimate the impact on smoking and SLT prevalence and attributable deaths of policies implemented between 1993 and 2018 and the impact of stronger future policies implemented in 2018 and maintained through 2060.

Results: SimSmoke generally reflects trends in exclusive cigarette use from the TUS-CPS and the Behavioral Risk Factor Surveillance System (BRFSS), but underestimated the increase in SLT prevalence in recent years. SimSmoke projects that policies implemented between 1993 and 2018 reduced male and female cigarette use by $23.7 \%$ and $23.0 \%$, and male and female SLT use by $4.9 \%$ by 2018 , averting 9018 tobacco-attributable deaths by 2018, increasing to 89547 by 2060 . The largest reductions in cigarette and SLT use were attributed to cigarette price increases. Strengthening tobacco control policies could reduce smoking prevalence by $41 \%$ and $40 \%$, and reduce SLT prevalence by $33 \%$ and $25 \%$ for males and females by 2060 .

Conclusion: Our results suggest that cigarette-oriented policies were effective in reducing SLT use but have been less successful in recent years. Future use rates can be further reduced through more restrictive statewide policies, which also target non-combustible nicotine products.

Keywords: Smokeless Tobacco, Cigarette, Tobacco Policy, Simulation Model, Kentucky

Copyright: @ 2022 The Author(s); Published by Kerman University of Medical Sciences. This is an open-access article distributed under the terms of the Creative Commons Attribution License (https://creativecommons.org/licenses/ by/4.0), which permits unrestricted use, distribution, and reproduction in any medium, provided the original work is properly cited.

Citation: Sánchez-Romero LM, Yuan Z, Li Y, Levy DT. The Kentucky SimSmoke tobacco control policy model of smokeless tobacco and cigarette use. Int J Health Policy Manag. 2022;11(5):592-609. doi:10.34172/ijhpm.2020.187
\end{abstract}

Article History:

Received: 30 October 2019 Accepted: 26 September 2020 ePublished: 27 October 2020

\section{*Correspondence to:}

David T. Levy

Email: d1777@georgetown.edu

\section{Key Messages}

\section{Implications for policy makers}

- Kentucky has relatively weak policies, and stronger tobacco control policies are needed.

- In particular, policies in Kentucky need to be directed at cigarette use, including higher cigarette taxes, comprehensive smoke-free air laws, and media campaigns

- Marketing restrictions, media campaigns and youth access restrictions should also be directed at smokeless tobacco (SLT) use.

- The tobacco industry should be monitored.

\section{Implications for the public}

While the landscape for nicotine delivery products has dramatically changed in the last ten years, some lessons can be gleaned from our results. First, with cigarettes as the dominant form of nicotine delivery, cigarette-oriented policies are an effective means of reducing the use of nicotine delivery products. Well targeted policies and regulations, such as cigarette and smokeless tobacco (SLT) tax increases and media campaigns, will be needed to achieve the national Healthy People 2020 objective of less than 1\% SLT use. However, with SLT use increasing in recent years, policies directed at SLT may also play a role. With cigarette manufacturers having acquired major SLT firms, it is important to monitor the role of the cigarette industry, as it has strong incentives to protect the high profits from cigarettes. Their incentives are to encourage dual use rather than having individuals switch to SLTs or quit all tobacco use. Similar implications may be expected for e-cigarettes. 


\section{Background}

Kentucky has one of the highest rates of cigarette smoking prevalence in the United States at about $24.6 \%$ in 2017, ${ }^{1}$ compared to $14.0 \%$ for the United States as a whole. ${ }^{2}$ Kentucky also has some of the weakest state-level tobacco control policies, ${ }^{3,4}$ with tax rates among the lowest of all states, limited smoke-free legislation in some cities, and tobacco control spending (less than $10 \%$ of the CDC [Centers for Disease Control and Prevention] recommended minimum) ranked among the lowest $25 \%$ of states in $2018 .{ }^{5}$ In addition to high smoking prevalence, Kentucky also has a high rate of smokeless tobacco (SLT) prevalence. The Behavioral Risk Factor Surveillance System (BRFSS) found that Kentucky SLT prevalence was 7.6\% (almost 14\% among males) ${ }^{1}$ in 2017 compared to the $4.1 \%$ national average. ${ }^{6-8}$ Also, any past-30 day use of SLT products among Kentucky high schoolers was estimated at $10.6 \%$ in 2017 , with the national average rate at $5.5 \%{ }^{9}$

While much of SLT use takes the traditional forms of snuff and chewing tobacco, new forms of smokeless, such as different varieties of snus and other oral tobacco products, have also become available in recent years. ${ }^{10,11}$ With lower health risks than cigarettes, ${ }^{12-14}$ these products may reduce the harms associated with cigarettes. Their impact will depend on the relationship of SLT use to smoking. ${ }^{15}$ If SLT use reduces smoking initiation or helps smokers who would not have otherwise quit to either quit or entirely switch to SLTs, then public health improves. On the other hand, SLT use may encourage youths to take up tobacco use or discourage smokers who would have otherwise quit from actually quitting, and thus have a harmful public health impact. In particular, smokeless can act as a complementary product to smoking and help maintain smoking through dual use. While we focus on SLT use, similar concerns arise with e-cigarette use. ${ }^{15}$ With a shorter history than SLT use, less is known about e-cigarette use. A better understanding of patterns of SLT use, especially among youth and young adults, and of the effects of tobacco control policies on SLT use may be helpful in understanding the public health impact of e-cigarette vis-à-vis cigarette use and the potential impact of policies on that use.

The Kentucky SimSmoke tobacco control simulation model is used to examine the impact of past and stronger future tobacco control policies on Kentucky's tobacco users. This paper updates a previous version of Kentucky SimSmoke (1993-2006) $)^{3}$ to consider smoking prevalence through 2018 and incorporate exclusive and dual SLT use. The new model distinguishes exclusive and dual SLT use and considers the impact of implementing individual and combined policies on smoking and SLT prevalence and tobacco-attributable deaths in Kentucky.

\section{Methods}

The Kentucky SimSmoke, a discrete first-order Markov model, starts with the 1993 population by age and gender. The population is distributed into seven smoking categories: never tobacco users, current and former exclusive smokers, current and former exclusive SLT users, and current and former dual users. Over time, cigarette and SLT users at each age change through four principal modules: population, tobacco use, tobacco-attributable deaths, and tobacco regulation policies. The model is based on a previously developed model for the United States. ${ }^{16}$ Further discussion of the new Kentucky model is found in a longer supplementary report. ${ }^{17}$

\section{Population}

The population evolves through births, deaths and migration. Kentucky population data was obtained from the Census Bureau for 1993 to 2015, ${ }^{18}$ with projections through 2040. ${ }^{19}$ SimSmoke uses the actual population for ages 0-14, with the population for 2040-2060 linearly extrapolated from 20302040. At later ages, the population evolves via mortality and migration rates, ${ }^{20}$ and was adjusted to within $7 \%$ of 2015 Kentucky population estimates.

\section{Tobacco Use}

Individuals evolve from never tobacco users to current exclusive smokers, exclusive SLT, or dual users through cigarette, SLT, and dual initiation. Before age 14, the population is considered to consist entirely of never smokers. From age 15, a percentage of each age group transitions to current tobacco users through smoking and SLT initiation rates. Current exclusive smokers, exclusive SLT users, or dual users may quit and thereby become former users. Former exclusive smokers through cessation may return to smoking through relapse and similarly for former exclusive SLT users and former dual users. Former dual users do not relapse to exclusive tobacco users. A discrete time, first-order Markov process is employed to project smoking, SLT use, and dual use rates through initiation, cessation, and relapse.

Baseline estimates of exclusive smoking, exclusive SLT, and dual use status by age and gender were obtained from the nationally-representative 1992/1993 Tobacco Use Supplement of the Current Population Survey (TUS-CPS). ${ }^{21}$ Current smoker is defined as having smoked more than 100 cigarettes in his/her lifetime and currently smoking either daily or some days. A question on SLT "use on a regular basis" is used to distinguish SLT users. Dual use is defined as those meeting the respective definitions of current smokers and SLT users. Due to the small female dual use prevalence $(<0.1 \%)$, we do not consider this group. Former use is defined as those meeting their respective definitions of tobacco-use, but reporting no current use, and they are stratified into quit-years groups $(<1$, $1-2,3-4,5-9,10-15,16+$ years). Since former smokers were not distinguished by former exclusive users and former dual users, they were estimated by multiplying former smoking prevalence by years quit by the ratio of current exclusive smokers or current dual users to total current smokers.

The TUS-CPS does not provide sufficient information to distinguish initiation, cessation, and switching rates between SLT use and cigarettes, and the evidence on initiation and early transitions to SLT use is mixed. ${ }^{22-26}$ To measure initiation while incorporating cessation and switching, we calculate net initiation as the difference between 1993 prevalence at the current age and previous age. Since studies indicate limited switching between SLT use and cigarettes except at younger ages, ${ }^{27-29}$ we allow switching only via net initiation. Based on 
cigarette and SLT use peak rates, we allowed cigarette and SLT net initiation through age 27 for males and 34 for females.

Tobacco cessation is explicitly modeled after the last age of net initiation. Smoker quit rates were obtained from the $1992 / 1993$ TUS-CPS, measured as those who quit in the last year, but not the last three months. ${ }^{30}$ Because data were not available to estimate quit rates for exclusive SLT and dual users, we assumed that dual and exclusive SLT users quit and relapse rates by years quit are the same as for smokers ${ }^{31-34}$ based on findings that quit rates are at least as high among SLT and dual users as for cigarette users..$^{27-29,35,36}$ Age, gender, and quit year-specific relapse rates are obtained from the US SimSmoke. ${ }^{31-34}$

\section{Tobacco-Attributable Deaths}

Mortality rates were developed for each age, gender, and tobacco use category using the relative risks and prevalence rates of the categories and overall mortality by age and gender. Data on mortality relative risk for current and former smokers by age and gender were from the Cancer Prevention Study II. ${ }^{33,37,38}$ We assigned the same risks to exclusive cigarette and dual users, with risks declining for both former user categories with years quit. ${ }^{33,37,38}$ SLT users were assigned an overall mortality relative risk of 1.15 based on results from a large-scale US study. ${ }^{13}$

Tobacco-attributable deaths were calculated as the number of users in each current and former smoking category multiplied by their excess mortality risk (ie, mortality rate of each current and former tobacco use group minus never smoker mortality rate) and then summed.

\section{Policies}

SimSmoke begins with policies at their 1993 levels and then incorporates the effect of policy changes between 1993 and 2018. SimSmoke includes tobacco prices (taxes), smoke-free air laws, mass media campaigns, marketing restrictions, health warnings, cessation treatment policies, and youth access. Table 1 shows the policy descriptions and effect sizes. Most policies are modeled as having direct reductions in prevalence rates (immediate impact) with the effect growing over time from changes in the initiation and cessation rates. Each policy effect size is estimated in terms of the percentage change relative to initial rates. All policy effect sizes are multiplicatively applied. Policy levels are based on the specific characteristics of each policy (eg, size of the tax, expenditures on media campaigns, coverage of smoke-free-air laws). Policy levels defined in Table 1, are based on those in effect in January of the corresponding year.

Cigarette and SLT price effects depend on price elasticities, prices through the tracking period, and tax changes in future years. ${ }^{39}$ Price elasticities are based on demand studies, which report similar elasticities for SLT and cigarettes. ${ }^{40-42}$ Cigarette prices are based on average Kentucky cigarette retail prices (including generics) ${ }^{43}$ from 1993 to 2018 . SLT prices, the weighted average of the prices of chewing tobacco, snuff, and snus, are estimated by manufacturer prices, state and federal taxes, and wholesale and retail mark-ups. ${ }^{44}$ We adjusted post2015 cigarettes and SLT prices by their respective state tax increases and inflation using the consumer price index. ${ }^{45}$ Price effects were weighted (75\% cigarette, 25\% SLT) for dual users. ${ }^{17}$ Federal and Kentucky cigarette excise taxes were increased in 2000, 2002, 2005, and 2009. Federal and Kentucky SLT taxes were increased in 2000, 2005, 2009, and 2010 , then slightly decreased in $2013 .^{17}$

Smoke-free air laws consider the existence and enforcement of worksite, restaurant, pubs and bars, and other public place bans. ${ }^{46}$ The policy effect sizes (shown in Table 1) on exclusive SLT and dual use are assigned $25 \%$ of cigarette use. ${ }^{42}$ Before 2003, Kentucky had no significant state smoke-free air laws, but since then, local restaurants, bars, and indoor workplace bans were implemented to cover about one-third of the state population. ${ }^{47}$ Based on estimates from the US model, ${ }^{16}$ we estimated 80\% compliance for all users in 1993-2018.

SimSmoke considers tobacco control expenditures, which are usually for media campaigns. ${ }^{48}$ Based on evaluation studies of SLT-oriented media campaigns, ${ }^{42}$ the policy effect size for exclusive SLT use is $50 \%$ less than for exclusive and dual cigarette users. The levels are distinguished as high, medium, or low. ${ }^{49}$ Based on state expenditures, ${ }^{50}$ this policy level is set at low in 1993-1999, medium in 2000-2002, and low since 2003.

Marketing restrictions are distinguished as high, medium, or low levels. In Kentucky, the policy was assigned a low level in all years with $80 \%$ exposure. The effect sizes for smokers and SLT users are the same.

Health warnings depend on the size, location and whether they are graphic. ${ }^{51}$ Warnings on cigarette packages at a minimal level $(<30 \%$ of the package) have not changed since first implemented in 1966. SLT package warnings were also at a minimum level until 2009. Since 2010, SLT packages have been required to display large text warnings covering at least $30 \%$ of two principal sides, raising the level to moderate. ${ }^{42,52}$

Cessation treatment policies include the implementation and enforcement of four sub-policies: pharmacotherapy (PT) availability, financial coverage of treatments, quitlines, and brief interventions. ${ }^{53}$ Reviews of SLT cessation trials find mixed effects for pharmacotherapies, although slightly stronger effects for behavioral interventions. ${ }^{42}$ Nonetheless, SLT users utilize these resources at low rates. ${ }^{54}$ We set a $50 \%$ and $25 \%$ reduction of this policy effect for SLT and dual users, respectively, from the effect for smokers. Based on data from private and other healthcare program coverage in Kentucky, we estimate that $30 \%$ of the population is covered for PT and behavioral therapy from 1993 to 2009 , increasing to $35 \%$ in 2010 with Medicaid coverage, and to $50 \%$ in 2014 with the Affordable Care Act. About 40\% of smokers in all years were receiving brief interventions (asking about smoking, advising to quit and recommending effective cessation treatments) at appropriate levels from their healthcare providers. ${ }^{55}$ We also classify Kentucky as having no effective quitline until 2005, when the Kentucky Tobacco Quitline was implemented. ${ }^{56}$

Strongly enforced and publicized youth access laws yield a larger reduction in youth smoking initiation for 10-15 yearolds than for 16-17 year-olds, and is enhanced by vending machines and self-service bans. ${ }^{57}$ Studies report lower compliance rates for SLT use than for cigarettes sales, ${ }^{58,59}$ and 
Table 1. Policy Inputs for Cigarette and Smokeless Tobacco SimSmoke

\begin{tabular}{|c|c|c|c|}
\hline Policy & Description & Cigarette Effect Size* & SLT Effect Size \\
\hline \multicolumn{4}{|l|}{ Tax policy } \\
\hline Cigarette and SLT price & $\begin{array}{l}\text { The effect of taxes is directly incorporated through average US } \\
\text { price (including generics), with separate prices for cigarettes } \\
\text { and SLT. The price elasticity (by age) is used to convert the } \\
\text { price into effect sizes. The dual price computed as } 4 / 5 \text { of the } \\
\text { cigarette price }+1 / 5 \text { SLT price. }\end{array}$ & $\begin{array}{l}\text { Elasticities } \\
-0.4 \text { ages } 10-17 \\
-0.3 \text { ages } 18-24 \\
-0.2 \text { ages } 25-34 \\
-0.1 \text { ages } 35-64 \\
-0.2 \text { ages } 65 \text { and above }\end{array}$ & $\begin{array}{l}\text { Elasticities (exclusive } \\
\text { SLT only) } \\
-0.4 \text { ages } 10-17 \\
-0.3 \text { ages } 18-24 \\
-0.2 \text { ages } 25 \text { and above }\end{array}$ \\
\hline \multicolumn{4}{|l|}{ Smoke-free air laws } \\
\hline $\begin{array}{l}\text { Worksite smoking ban, well- } \\
\text { enforced }\end{array}$ & $\begin{array}{l}\text { Ban in all indoor worksites in all areas, with strong public } \\
\text { acceptance and enforcement of laws (reduced by } 1 / 3 \text { if } \\
\text { allowed in ventilated areas and by } 2 / 3 \text { if allowed in common } \\
\text { areas. }\end{array}$ & $-6 \%$ & $\begin{array}{l}25 \% \text { smoking effect } \\
\text { size for exclusive SLT } \\
\text { users and dual users }\end{array}$ \\
\hline Restaurant smoking ban & $\begin{array}{l}\text { Ban in all indoor restaurants in all areas (reduced by half if } \\
\text { partial). }\end{array}$ & $-2 \%$ & \\
\hline Bars smoking ban & Ban in all indoor bars in all areas (reduced by half if partial). & $-1 \%$ & \\
\hline Other places bans & $\begin{array}{l}\text { Ban in } 3 \text { of } 4 \text { of government buildings, retail stores, public } \\
\text { transportation and elevators. }\end{array}$ & $-1 \%$ & \\
\hline Compliance & The government enforces and publicizes laws. & $\begin{array}{l}\text { Effects reduced by } 50 \% \text { if no } \\
\text { compliance }\end{array}$ & Same \\
\hline \multicolumn{4}{|l|}{ Media campaigns } \\
\hline $\begin{array}{l}\text { Highly publicized media } \\
\text { campaign }\end{array}$ & $\begin{array}{l}\text { Campaign publicized heavily on TV and at least some other } \\
\text { media, with a social marketing approach. }\end{array}$ & $-6.50 \%$ & \multirow{3}{*}{$\begin{array}{l}50 \% \text { smoking effect } \\
\text { size for exclusive SLT } \\
\text { users; } 100 \% \text { smoking } \\
\text { effect size for dual } \\
\text { users }\end{array}$} \\
\hline $\begin{array}{l}\text { Moderately publicized media } \\
\text { campaign }\end{array}$ & $\begin{array}{l}\text { Campaign publicized sporadically on TV and at least some } \\
\text { other media. }\end{array}$ & $-3.25 \%$ & \\
\hline $\begin{array}{l}\text { Low publicity media } \\
\text { campaign }\end{array}$ & $\begin{array}{l}\text { Campaign publicized only sporadically in newspapers, } \\
\text { billboards or some other media. }\end{array}$ & $-1.63 \%$ & \\
\hline \multicolumn{4}{|l|}{ Marketing restrictions } \\
\hline $\begin{array}{l}\text { Comprehensive marketing } \\
\text { ban }\end{array}$ & $\begin{array}{l}\text { Ban is applied to television, radio, print, billboard, in- } \\
\text { store displays, sponsorships and free samples (all indirect } \\
\text { marketing). }\end{array}$ & $\begin{array}{l}-5 \% \text { prevalence, }-8 \% \\
\text { initiation, }+4 \% \text { cessation }\end{array}$ & \multirow{4}{*}{$\begin{array}{l}\text { Same (100\%) smoking } \\
\text { effect size for exclusive } \\
\text { SLT users and dual } \\
\text { users }\end{array}$} \\
\hline Total advertising ban & $\begin{array}{l}\text { Ban is applied to all media television, radio, print, billboard, } \\
\text { plus one indirect marketing. }\end{array}$ & $\begin{array}{l}-3 \% \text { prevalence, }-4 \% \\
\text { initiation, }+2 \% \text { cessation }\end{array}$ & \\
\hline Weak advertising ban & Ban is applied to some of television, radio, print, billboard. & $\begin{array}{l}-1 \% \text { in prevalence and } \\
\text { initiation only }\end{array}$ & \\
\hline Compliance & Based on exposure to advertisements. & $\begin{array}{l}\text { Effects reduced by } 50 \% \text { if } 0 \\
\text { exposure }\end{array}$ & \\
\hline \multicolumn{4}{|l|}{ Health warnings } \\
\hline Strong & $\begin{array}{l}\text { Labels are large, bold and graphic and at least } 30 \% \text { of the } \\
\text { package. }\end{array}$ & $\begin{array}{l}-4 \% \text { prevalence, }-6 \% \\
\text { initiation, }+10 \% \text { cessation }\end{array}$ & \multirow{3}{*}{$\begin{array}{l}\text { Same (100\%) smoking } \\
\text { effect size for exclusive } \\
\text { SLT users and dual } \\
\text { users }\end{array}$} \\
\hline Moderate & Laws cover $1 / 3$ of the package, not bold or graphic. & $\begin{array}{l}-2 \% \text { in prevalence } \& \\
\text { initiation, }+4 \text { cessation }\end{array}$ & \\
\hline Weak & Laws cover less than $1 / 3$ of the package, not bold or graphic. & $\begin{array}{l}1 \% \text { prevalence } \& \text { initiation, } \\
+2 \% \text { cessation }\end{array}$ & \\
\hline \multicolumn{4}{|l|}{ Cessation treatment policies } \\
\hline $\begin{array}{l}\text { Availability of } \\
\text { pharmacotherapies }\end{array}$ & Legality of NRT, Wellbutrin and varenicline. & $\begin{array}{l}-1 \% \text { prevalence, }+4 \% \\
\text { cessation }\end{array}$ & \multirow{5}{*}{$\begin{array}{l}50 \% \text { smoking effect } \\
\text { size for exclusive SLT } \\
\text { users; } 75 \% \text { smoking } \\
\text { effect size for dual } \\
\text { users }\end{array}$} \\
\hline Proactive quitline & $\begin{array}{l}\text { A proactive quitline with publicity through the media } \\
\text { campaign with no cost NRT. }\end{array}$ & $\begin{array}{l}-1 \% \text { prevalence, }+6 \% \\
\text { cessation }\end{array}$ & \\
\hline Treatment coverage & Payments to cover PT and behavioral cessation treatment. & $\begin{array}{l}-2.25 \% \text { prevalence, }+8 \% \\
\text { cessation }\end{array}$ & \\
\hline $\begin{array}{l}\text { Brief healthcare provider } \\
\text { interventions }\end{array}$ & $\begin{array}{l}\text { Asking about smoking, advising to quit and recommending } \\
\text { effective cessation treatments. }\end{array}$ & $\begin{array}{l}-1 \% \text { prevalence, }+4 \% \\
\text { cessation }\end{array}$ & \\
\hline All of the above & $\begin{array}{l}\text { Complete availability and reimbursement of pharmaco- and } \\
\text { behavioral treatments, quitlines, and brief interventions. }\end{array}$ & $\begin{array}{l}-5.74 \% \text { prevalence, }+29.44 \% \\
\text { cessation }\end{array}$ & \\
\hline
\end{tabular}


Table 1. Continued

\begin{tabular}{|c|c|c|c|}
\hline Policy & Description & Cigarette Effect Size* & SLT Effect Size \\
\hline \multicolumn{4}{|l|}{ Youth access policies } \\
\hline $\begin{array}{l}\text { Strongly enforced \& } \\
\text { publicized }\end{array}$ & $\begin{array}{l}\text { Compliance checks are conducted } 4 \text { times per year per outlet, } \\
\text { penalties are potent and enforced, and with heavy publicity. }\end{array}$ & $\begin{array}{l}-16 \% \text { initiation for ages } 16- \\
17 \text { and }-24 \% \text { ages }<16\end{array}$ & \multirow{6}{*}{$\begin{array}{l}50 \% \text { smoking effect } \\
\text { size for exclusive SLT } \\
\text { users; } 100 \% \text { smoking } \\
\text { effect size for dual } \\
\text { users }\end{array}$} \\
\hline Well-enforced & $\begin{array}{l}\text { Compliance checks are conducted regularly, penalties are } \\
\text { potent, and publicity and merchant training are included. }\end{array}$ & $\begin{array}{l}8 \% \text { initiation for ages } 16-17 \\
\text { and }-12 \% \text { ages }<16\end{array}$ & \\
\hline Low enforcement & $\begin{array}{l}\text { Compliance checks are conducted sporadically, penalties are } \\
\text { weak. }\end{array}$ & $\begin{array}{l}2 \% \text { initiation for ages } 16-17 \\
\text { and }-4 \% \text { ages }<16\end{array}$ & \\
\hline Vending machine ban & Total ban. & $\begin{array}{l}\text { Enforcement effects increase } \\
\text { by } 8 \%\end{array}$ & \\
\hline Self-service ban & Total ban. & $\begin{array}{l}\text { Enforcement effects increase } \\
\text { by } 4 \%\end{array}$ & \\
\hline Publicity & $\begin{array}{l}\text { Community based and merchant publicity campaigns directed } \\
\text { at youths. }\end{array}$ & $\begin{array}{l}\text { Enforcement effects increase } \\
\text { by } 10 \%\end{array}$ & \\
\hline
\end{tabular}

Abbreviations: SLT, smokeless tobacco; NRT, nicotine replacement therapy; PT, pharmacotherapy.

* Unless otherwise indicated, the effects are on prevalence in the first year, and on initiation and cessation during the years that the policy is in effect. The effect sizes are based on articles referenced in the text.

that tobacco youth access laws weakly affect youth SLT use. ${ }^{60,61}$ We assign SLT users 50\% and dual users $100 \%$ of the youth access policy cigarette effect sizes. Self-service and vending machine restrictions are set at 0\% until 1997, 50\% 1998-2008, and $100 \%$ since 2009. Enforcement was weak in 1997-1998 and increased to moderate since 1998.

\section{Calibration and Validation}

To calibrate SimSmoke, we compared its predictions of adult smoking and SLT prevalence by age and gender to corresponding estimates from the TUS-CPS data for 1996 and 1999. Based on these comparisons, we adjusted the first-year smoking cessation rates downward for ages 24-35 and upward for ages 55 and above.

To validate the model, we compared SimSmoke predictions considering all policies implemented from 1993 to 2017 to rates of exclusive cigarette, exclusive SLT, and dual use from the 2002/2003, 2006/2007, 2010/2011 and 2014/2015 TUSCPS survey. Results were also validated using gender-specific estimates from the BRFSS for overall smokers (exclusive cigarette and dual users) from 1996 to 2017. We performed a separate validation for total SLT (exclusive SLT and dual users) from 2011-2016. We also considered confidence intervals from each of the surveys.

\section{Assessing the Effect of Tobacco Control Policies}

Upon validating the model, we estimate the effect of three policy scenarios (status-quo, counterfactual, and future) for their impacts on exclusive cigarette, exclusive SLT, and dual user prevalence and tobacco-attributable deaths. The status quo scenario incorporates policies implemented from 1993 to 2018. The counterfactual scenario represents trends in the absence of any policy change and is programmed with all policies at their 1993 levels. We estimate the impact of policies through 2018 by calculating the relative difference between the counterfactual and status-quo scenarios. The contribution of an individual policy is based on programming SimSmoke changes through 2018 on the selected policy with other policies at the 1993 level. The effect of individual policies is measured relative to the summed effects of all individual policies.

We estimate the potential impact of future policies on tobacco prevalence by increasing all policies to their highest levels as shown in Table 1. The effects are presented relative to the status quo level in the same year for smoking and SLT prevalence and deaths averted.

For the effect of past and future policies, we also estimate lower and upper credible ranges of the policy effect sizes. Credible ranges are based on the potential minimum and maximum effect of the policy based on reported effects from the literature review. ${ }^{62}$ For cigarettes, they are modeling as $+/$ $50 \%$ of the original policy estimated effect, except $+/-25 \%$ for taxes. For SLT estimates, we use $+/-75 \%$ and $+/-50 \%$ for taxes.

\section{Results}

Model Validation

Validation comparing SimSmoke estimates to the TUS-CPS are in Table 2. For the total adult population, SimSmoke predicts a decrease in exclusive male (female) cigarette prevalence from $34.2 \%(29.5 \%)$ in 1993 to $21.2 \%(21.2 \%)$ in 2015 compared to a decrease from $34.6 \%(29.5 \%)$ to $20.8 \%$ (19.5\%) in 2015 from the TUS-CPS. Estimated SimSmoke prevalence from $2002 / 20032006 / 2007$ and 2010/2011 fell within the 95\% confidence intervals (CIs) in TUS-CPS data. For male dual users, SimSmoke predicts a decrease in prevalence from $2.1 \%$ in 1993 to $1.6 \%$ in 2015 (22\% relative reduction), while TUSCPS show a decrease from $2.0 \%$ in 1993 to $1.1 \%$ in 2015 (45\% relative reduction), but dual use increases between 2007 $(0.9 \%)$ and 2010 (1.3\%). Dual user predictions for all years fell within the $95 \%$ confidence intervals of the reported data.

SimSmoke predicts male exclusive SLT prevalence to continuously decline by $23 \%$ from $7.1 \%$ to $5.4 \%$ from 1993 to 2015, while TUS-CPS reports a reduction from $6.8 \%$ to $3.5 \%$ from 1993 to 2007 , but a $35 \%$ increase from $3.5 \%$ to $4.7 \%$ between 2007 and 2015. Validation by age and gender also perform relatively well (see Supplementary Report ${ }^{17}$ ). 
Sánchez-Romero et al

Table 2. Validation: Exclusive Cigarette, SLT, Dual Use, SimSmoke Projections vs. TUS-CPS, by Age and Gender, 1993-2015

\begin{tabular}{|c|c|c|c|c|c|c|c|c|c|}
\hline \multicolumn{10}{|c|}{ Male Exclusive Smokers } \\
\hline Ages & & 1993 & 2002 & 1993-2002* & 2007 & 2010 & 2015 & 2002-2015* & 1993-2015* \\
\hline \multirow[t]{3}{*}{$18+$} & SimSmoke & $34.2 \%$ & $27.9 \%$ & $-18.6 \%$ & $25.3 \%$ & $22.7 \%$ & $21.2 \%$ & $-24.1 \%$ & $-38 \%$ \\
\hline & TUS-CPS & $34.6 \%$ & $27.6 \%$ & $-20.3 \%$ & $28.4 \%$ & $23.2 \%$ & $20.8 \%$ & $-24.8 \%$ & $-40 \%$ \\
\hline & $95 \% \mathrm{Cl}$ & $(31.8 \%, 37.5 \%)$ & $(24.9 \%, 30.4 \%)$ & & $(23.9 \%, 32.9 \%)$ & $(20.8 \%, 25.7 \%)$ & $(18.5 \%, 23.3 \%)$ & & \\
\hline \multirow[t]{3}{*}{ 18-24 } & SimSmoke & $31.5 \%$ & $23.7 \%$ & $-24.7 \%$ & $21.6 \%$ & $20.0 \%$ & $19.9 \%$ & $-16.3 \%$ & $-37 \%$ \\
\hline & TUS-CPS & $30.9 \%$ & $43.8 \%$ & $41.9 \%$ & $27.5 \%$ & $21.7 \%$ & $21.0 \%$ & $-52.1 \%$ & $-32 \%$ \\
\hline & $95 \% \mathrm{Cl}$ & $(21.1 \%, 40.7 \%)$ & $(32.8 \%, 54.9 \%)$ & & $(11.4 \%, 43.5 \%)$ & $(14.6 \%, 30.8 \%)$ & $(12.7 \%, 32.6 \%)$ & & \\
\hline \multirow[t]{2}{*}{$25-44$} & SimSmoke & $38.0 \%$ & $32.0 \%$ & $-15.9 \%$ & $28.8 \%$ & $25.7 \%$ & $24.2 \%$ & $-24.2 \%$ & $-36 \%$ \\
\hline & $95 \% \mathrm{Cl}$ & $(34.0 \%, 42.6 \%)$ & $(27.6 \%, 37.0 \%)$ & & $(25.1 \%, 40.7 \%)$ & $(22.3 \%, 30.8 \%)$ & $(17.6 \%, 26.2 \%)$ & & \\
\hline \multirow[t]{3}{*}{$45-64$} & SimSmoke & $36.7 \%$ & $29.6 \%$ & $-19.3 \%$ & $26.6 \%$ & $23.9 \%$ & $21.7 \%$ & $-26.5 \%$ & $-41 \%$ \\
\hline & TUS-CPS & $37.1 \%$ & $27.8 \%$ & $-25.1 \%$ & $28.9 \%$ & $26.1 \%$ & $25.8 \%$ & $-7.2 \%$ & $-30 \%$ \\
\hline & $95 \% \mathrm{Cl}$ & $(31.8 \%, 42.5 \%)$ & $(23.2 \%, 32.4 \%)$ & & $(21.6 \%, 36.3 \%)$ & $(22.2 \%, 30.4 \%)$ & $(21.9 \%, 30.1 \%)$ & & \\
\hline \multirow[t]{3}{*}{$65+$} & SimSmoke & $21.3 \%$ & $17.0 \%$ & $-19.9 \%$ & $16.5 \%$ & $15.4 \%$ & $14.8 \%$ & $-13.0 \%$ & $-30 \%$ \\
\hline & TUS-CPS & $22.2 \%$ & $11.1 \%$ & $-50.2 \%$ & $18.6 \%$ & $11.7 \%$ & $11.3 \%$ & $2.4 \%$ & $-49 \%$ \\
\hline & $95 \% \mathrm{Cl}$ & $(16.1 \%, 28.3 \%)$ & $(6.6 \%, 15.5 \%)$ & & $(9.5 \%, 27.7 \%)$ & $(8.0 \%, 16.7 \%)$ & $(8.1 \%, 15.7 \%)$ & & \\
\hline \multicolumn{10}{|c|}{ Female Exclusive Smokers } \\
\hline \multirow[t]{3}{*}{$18+$} & SimSmoke & $29.5 \%$ & $25.9 \%$ & $-12.3 \%$ & $24.2 \%$ & $22.2 \%$ & $21.2 \%$ & $-18.2 \%$ & $-28 \%$ \\
\hline & TUS-CPS & $29.5 \%$ & $24.7 \%$ & $-16.5 \%$ & $26.7 \%$ & $23.5 \%$ & $19.5 \%$ & $-20.9 \%$ & $-34 \%$ \\
\hline & $95 \% \mathrm{Cl}$ & $(27.2 \%, 31.8 \%)$ & $(22.4 \%, 27.0 \%)$ & & $(23.1 \%, 30.4 \%)$ & $(21.4 \%, 25.7 \%)$ & $(17.5 \%, 21.8 \%)$ & & \\
\hline \multirow[t]{3}{*}{$18-24$} & SimSmoke & $34.9 \%$ & $27.1 \%$ & $-22.2 \%$ & $25.3 \%$ & $23.4 \%$ & $23.4 \%$ & $-13.8 \%$ & $-33 \%$ \\
\hline & TUS-CPS & $38.5 \%$ & $37.8 \%$ & $-1.7 \%$ & $29.7 \%$ & $30.4 \%$ & $16.7 \%$ & $-55.9 \%$ & $-57 \%$ \\
\hline & $95 \% \mathrm{Cl}$ & $(30.6 \%, 46.3 \%)$ & $(29.1 \%, 46.5 \%)$ & & $(17.9 \%, 41.6 \%)$ & $(22.3 \%, 39.9 \%)$ & $(10.5 \%, 25.4 \%)$ & & \\
\hline \multirow[t]{3}{*}{ 25-44 } & SimSmoke & $36.1 \%$ & $32.8 \%$ & $-9.3 \%$ & $30.5 \%$ & $27.6 \%$ & $25.9 \%$ & $-20.9 \%$ & $-28 \%$ \\
\hline & TUS-CPS & $35.6 \%$ & $30.8 \%$ & $-13.5 \%$ & $32.8 \%$ & $26.9 \%$ & $24.4 \%$ & $-20.7 \%$ & $-31 \%$ \\
\hline & $95 \% \mathrm{Cl}$ & $(31.9 \%, 39.3 \%)$ & $(26.9 \%, 34.7 \%)$ & & $(26.1 \%, 39.6 \%)$ & $(23.2 \%, 30.8 \%)$ & $(20.5 \%, 28.9 \%)$ & & \\
\hline \multirow[t]{3}{*}{$45-64$} & SimSmoke & $29.2 \%$ & $26.1 \%$ & $-10.6 \%$ & $24.3 \%$ & $22.3 \%$ & $21.1 \%$ & $-19.1 \%$ & $-28 \%$ \\
\hline & TUS-CPS & $29.5 \%$ & $22.5 \%$ & $-23.6 \%$ & $28.5 \%$ & $26.9 \%$ & $23.6 \%$ & $4.7 \%$ & $-20 \%$ \\
\hline & $95 \% \mathrm{Cl}$ & $(25.1 \%, 34.0 \%)$ & $(18.8 \%, 26.3 \%)$ & & $(22.4 \%, 34.6 \%)$ & $(23.4 \%, 30.7 \%)$ & $(20.0 \%, 27.6 \%)$ & & \\
\hline \multirow[t]{3}{*}{$65+$} & SimSmoke & $12.4 \%$ & $11.5 \%$ & $-6.9 \%$ & $12.5 \%$ & $12.7 \%$ & $13.0 \%$ & $13.0 \%$ & $5 \%$ \\
\hline & TUS-CPS & $12.4 \%$ & $9.5 \%$ & $-23.4 \%$ & $11.5 \%$ & $10.8 \%$ & $9.0 \%$ & $-5.8 \%$ & $-28 \%$ \\
\hline & $95 \% \mathrm{Cl}$ & $(8.7 \%, 16.1 \%)$ & $(5.9 \%, 13.1 \%)$ & & $(5.5 \%, 17.4 \%)$ & $(8.0 \%, 14.6 \%)$ & $(6.4 \%, 12.4 \%)$ & & \\
\hline
\end{tabular}




\begin{tabular}{|c|c|c|c|c|c|c|c|c|c|}
\hline \multicolumn{10}{|c|}{ Male Dual Use } \\
\hline Ages & & 1993 & 2002 & 1993-2002* & 2007 & 2010 & 2015 & 2002-2015* & 1993-2015* \\
\hline \multirow[t]{3}{*}{$18+$} & SimSmoke & $2.1 \%$ & $1.8 \%$ & $-13.0 \%$ & $1.7 \%$ & $1.6 \%$ & $1.6 \%$ & $-10.7 \%$ & $-22 \%$ \\
\hline & TUS-CPS & $2.0 \%$ & $1.2 \%$ & $-39.6 \%$ & $0.9 \%$ & $1.3 \%$ & $1.1 \%$ & $-9.7 \%$ & $-45 \%$ \\
\hline & $95 \% \mathrm{Cl}$ & $(1.1 \%, 2.8 \%)$ & $(0.5 \%, 1.9 \%)$ & & $(0.0 \%, 1.9 \%)$ & $(0.8 \%, 2.1 \%)$ & $(0.6 \%, 1.9 \%)$ & & \\
\hline \multirow[t]{3}{*}{$18-24$} & SimSmoke & $3.4 \%$ & $2.1 \%$ & $-38.1 \%$ & $2.1 \%$ & $2.0 \%$ & $2.0 \%$ & $-3.6 \%$ & $-40 \%$ \\
\hline & TUS-CPS & $3.8 \%$ & $2.0 \%$ & $-46.9 \%$ & $0.0 \%$ & $2.1 \%$ & $4.8 \%$ & $139.9 \%$ & $27 \%$ \\
\hline & $95 \% \mathrm{Cl}$ & $(0.1 \%, 7.5 \%)$ & $(0.0 \%, 4.9 \%)$ & & $(0.0 \%, 0.0 \%)$ & $(0.6 \%, 7.2 \%)$ & $(1.7 \%, 13.3 \%)$ & & \\
\hline \multirow[t]{2}{*}{$25-44$} & SimSmoke & $2.5 \%$ & $2.7 \%$ & $10.1 \%$ & $2.5 \%$ & $2.2 \%$ & $2.0 \%$ & $-25.6 \%$ & $-18 \%$ \\
\hline & $95 \% \mathrm{Cl}$ & $(1.1 \%, 3.9 \%)$ & $(0.5 \%, 3.3 \%)$ & & $(-0.6 \%, 3.3 \%)$ & $(1.7 \%, 5.1 \%)$ & $(1.0 \%, 4.1 \%)$ & & \\
\hline \multirow[t]{3}{*}{$45-64$} & SimSmoke & $0.7 \%$ & $1.0 \%$ & $40.3 \%$ & $1.3 \%$ & $1.5 \%$ & $1.6 \%$ & $69.6 \%$ & $138 \%$ \\
\hline & TUS-CPS & $0.5 \%$ & $0.6 \%$ & $17.0 \%$ & $1.1 \%$ & $0.2 \%$ & $0.5 \%$ & $-26.1 \%$ & $-14 \%$ \\
\hline & $95 \% \mathrm{Cl}$ & $(-0.3 \%, 1.3 \%)$ & $(-0.2 \%, 1.4 \%)$ & & $(-0.6 \%, 2.7 \%)$ & $(0.0 \%, 1.3 \%)$ & $(0.1 \%, 1.7 \%)$ & & \\
\hline \multirow[t]{3}{*}{$65+$} & SimSmoke & $2.0 \%$ & $0.8 \%$ & $-61.2 \%$ & $0.5 \%$ & $0.3 \%$ & $0.4 \%$ & $-52.9 \%$ & $-82 \%$ \\
\hline & TUS-CPS & $2.1 \%$ & $0.4 \%$ & $-80.2 \%$ & $0.0 \%$ & $0.0 \%$ & $0.0 \%$ & $-100.0 \%$ & $-100 \%$ \\
\hline & $95 \% \mathrm{Cl}$ & $(0.0 \%, 4.1 \%)$ & $(0.0 \%, 1.2 \%)$ & & $(0.0 \%, 0.0 \%)$ & $(0.0 \%, 1.8 \%)$ & $(0.0 \%, 1.4 \%)$ & & \\
\hline \multicolumn{10}{|c|}{ Male Exclusive Smokeless Tobacco Use } \\
\hline \multirow[t]{3}{*}{$18+$} & SimSmoke & $7.1 \%$ & $6.1 \%$ & $-12.9 \%$ & $5.9 \%$ & $5.6 \%$ & $5.4 \%$ & $-12.2 \%$ & $-23 \%$ \\
\hline & TUS-CPS & $6.8 \%$ & $4.1 \%$ & $-39.0 \%$ & $3.5 \%$ & $4.0 \%$ & $4.7 \%$ & $14.3 \%$ & $-30 \%$ \\
\hline & $95 \% \mathrm{Cl}$ & $(5.3 \%, 8.3 \%)$ & $(2.9 \%, 5.4 \%)$ & & $(1.7 \%, 5.3 \%)$ & $(3.0 \%, 5.2 \%)$ & $(3.7 \%, 6.2 \%)$ & & \\
\hline \multirow[t]{3}{*}{$18-24$} & SimSmoke & $10.4 \%$ & $7.2 \%$ & $-30.9 \%$ & $7.6 \%$ & $7.8 \%$ & $7.9 \%$ & $10.3 \%$ & $-24 \%$ \\
\hline & TUS-CPS & $12.0 \%$ & $4.9 \%$ & $-59.4 \%$ & $0.0 \%$ & $4.1 \%$ & $4.8 \%$ & $-0.6 \%$ & $-60 \%$ \\
\hline & $95 \% \mathrm{Cl}$ & $(5.1 \%, 18.9 \%)$ & $(0.1 \%, 9.7 \%)$ & & $(0.0 \%, 0.0 \%)$ & $(1.6 \%, 10.1 \%)$ & $(1.7 \%, 13.3 \%)$ & & \\
\hline \multirow[t]{3}{*}{$25-44$} & SimSmoke & $6.9 \%$ & $7.0 \%$ & $1.5 \%$ & $6.6 \%$ & $6.2 \%$ & $5.8 \%$ & $-17.1 \%$ & $-16 \%$ \\
\hline & TUS-CPS & $6.7 \%$ & $4.8 \%$ & $-28.9 \%$ & $4.9 \%$ & $4.6 \%$ & $6.0 \%$ & $24.7 \%$ & $-11 \%$ \\
\hline & $95 \% \mathrm{Cl}$ & $(4.5 \%, 9.0 \%)$ & $(2.7 \%, 6.9 \%)$ & & $(1.3 \%, 8.5 \%)$ & $(3.0 \%, 7.1 \%)$ & $(3.9 \%, 9.0 \%)$ & & \\
\hline \multirow[t]{3}{*}{$45-64$} & SimSmoke & $5.1 \%$ & $5.1 \%$ & $-1.1 \%$ & $5.0 \%$ & $4.9 \%$ & $4.9 \%$ & $-3.8 \%$ & $-5 \%$ \\
\hline & TUS-CPS & $4.9 \%$ & $2.9 \%$ & $-41.2 \%$ & $2.0 \%$ & $3.0 \%$ & $4.3 \%$ & $52.2 \%$ & $-11 \%$ \\
\hline & $95 \% \mathrm{Cl}$ & $(2.5 \%, 7.2 \%)$ & $(1.1 \%, 4.6 \%)$ & & $(-0.3 \%, 4.3 \%)$ & $(1.7 \%, 5.0 \%)$ & $(2.8 \%, 6.7 \%)$ & & \\
\hline \multirow[t]{3}{*}{$65+$} & SimSmoke & $7.6 \%$ & $5.2 \%$ & $-32.0 \%$ & $4.4 \%$ & $4.0 \%$ & $3.8 \%$ & $-26.2 \%$ & $-50 \%$ \\
\hline & TUS-CPS & $7.9 \%$ & $5.0 \%$ & $-36.9 \%$ & $5.2 \%$ & $4.7 \%$ & $3.8 \%$ & $-24.5 \%$ & $-52 \%$ \\
\hline & $95 \% \mathrm{Cl}$ & $(3.9 \%, 11.9 \%)$ & $(1.9 \%, 8.1 \%)$ & & $(0.0 \%, 10.5 \%)$ & $(2.6 \%, 8.4 \%)$ & $(2.1 \%, 6.8 \%)$ & & \\
\hline
\end{tabular}

Abbreviations: SLT, smokeless tobacco; TUS-CPS, Tobacco Use Supplement of the Current Population Survey. 
In a validation against the BRFSS (see Table $3 c$ of Supplementary Report ${ }^{17}$ ), male (female) smokers from the BRFSS shows a $20.6 \%$ (24.3\%) relative reduction between 1996 and 2017 compared to a $38.2 \%$ (31.0\%) relative reduction predicted by SimSmoke. SimSmoke predictions fell within the BRFSS 95\% CIs before 2011 and lower than the intervals since 2011 for both genders. SLT use from the BRFSS shows a 7.1\% (33.3\%) relative increase between 2011 and 2016 compared to a $3.4 \%(13.0 \%)$ relative reduction predicted by SimSmoke, with all the SimSmoke predictions below the BRFSS 95\% CI lower bounds.

The Effect of Policies Implemented Through 2018
Results comparing the status-quo scenario (ie, policies implemented between 1993 and 2018) to the counterfactual scenario (ie, policies set at 1993 level) are in Table 3 for smoking and SLT prevalence and Table 4 for averted tobaccoattributable deaths.

Compared to the counterfactual, SimSmoke projects that exclusive cigarette prevalence was reduced in relative terms by $23.7 \%$ (with credible range of $16.7 \%, 30.4 \%$ ) for males and $23.0 \%(16.2 \%, 29.4 \%)$ for females by 2018 . The 2018 reduction for male dual users was $16.4 \%(11.6 \%, 21.2 \%)$ and for exclusive male SLT users was $4.9 \%(1.2 \%, 8.6 \%)$. By 2060 , the model projects a relative reduction of $32.7 \%$

Table 3. Prevalence of Cigarette/SLT/Dual Use in Each Policy Scenario* and the Relative Difference Compared with Counterfatural Scenario, ${ }^{* *} 1993-2060$

\begin{tabular}{|c|c|c|c|c|c|c|c|}
\hline Scenario & & 1993 & 2018 & 2040 & 2060 & $\begin{array}{l}\text { Relative Difference } \\
\text { Compared to } \\
\text { Counterfactual in } 2040\end{array}$ & $\begin{array}{c}\text { Relative Difference } \\
\text { Compared to Counterfactual } \\
\text { in } 2060\end{array}$ \\
\hline \multicolumn{8}{|c|}{ Male } \\
\hline \multirow{6}{*}{$\begin{array}{l}\text { Counter- } \\
\text { factual }\end{array}$} & $\mathrm{CIG}$ & $34.2 \%$ & $26.4 \%$ & $22.9 \%$ & $22.2 \%$ & - & - \\
\hline & Range & - & $(26.4 \%, 26.4 \%)$ & $(22.9 \%, 22.9 \%)$ & $(22.2 \%, 22.2 \%)$ & - & - \\
\hline & Dual & $2.1 \%$ & $1.9 \%$ & $1.7 \%$ & $1.6 \%$ & - & - \\
\hline & Range & - & $(1.9 \%, 1.9 \%)$ & $(1.7 \%, 1.7 \%)$ & $(1.6 \%, 1.6 \%)$ & - & - \\
\hline & SLT & $7.1 \%$ & $5.6 \%$ & $4.8 \%$ & $4.5 \%$ & - & - \\
\hline & Range & - & $(5.6 \%, 5.6 \%)$ & $(4.8 \%, 4.8 \%)$ & $(4.5 \%, 4.5 \%)$ & - & - \\
\hline \multirow{6}{*}{ Status quo } & $\mathrm{CIG}$ & $34.2 \%$ & $20.2 \%$ & $15.8 \%$ & $14.9 \%$ & $-30.7 \%$ & $-32.7 \%$ \\
\hline & Range & - & $(22.0 \%, 18.4 \%)$ & $(17.9 \%, 13.9 \%)$ & $(17.1 \%, 12.9 \%)$ & $(-21.6 \%,-39.2 \%)$ & $(-23.0 \%,-41.6 \%)$ \\
\hline & Dual & $2.1 \%$ & $1.6 \%$ & $1.4 \%$ & $1.3 \%$ & $-19.7 \%$ & $-20.2 \%$ \\
\hline & Range & - & $(1.7 \%, 1.5 \%)$ & $(1.5 \%, 1.3 \%)$ & $(1.4 \%, 1.2 \%)$ & $(-13.6 \%,-25.7 \%)$ & $(-14.0 \%,-26.6 \%)$ \\
\hline & SLT & $7.1 \%$ & $5.3 \%$ & $4.7 \%$ & $4.5 \%$ & $-1.5 \%$ & $0.9 \%$ \\
\hline & Range & - & $(5.5 \%, 5.1 \%)$ & $(4.9 \%, 4.6 \%)$ & $(4.6 \%, 4.4 \%)$ & $(1.1 \%,-4.3 \%)$ & $(2.8 \%,-1.4 \%)$ \\
\hline \multirow{6}{*}{ Price alone } & CIG & $34.2 \%$ & $21.8 \%$ & $17.7 \%$ & $16.9 \%$ & $-22.4 \%$ & $-23.7 \%$ \\
\hline & Range & - & $(22.9 \%, 20.8 \%)$ & $(18.9 \%, 16.6 \%)$ & $(18.1 \%, 15.8 \%)$ & $(-17.3 \%,-27.2 \%)$ & $(-18.4 \%,-28.8 \%)$ \\
\hline & Dual & $2.1 \%$ & $1.6 \%$ & $1.5 \%$ & $1.4 \%$ & $-14.9 \%$ & $-15.1 \%$ \\
\hline & Range & - & $(1.7 \%, 1.6 \%)$ & $(1.5 \%, 1.4 \%)$ & $(1.4 \%, 1.3 \%)$ & $(-11.3 \%,-18.4 \%)$ & $(-11.5 \%,-18.8 \%)$ \\
\hline & SLT & $7.1 \%$ & $5.4 \%$ & $4.9 \%$ & $4.7 \%$ & $2.6 \%$ & $5.4 \%$ \\
\hline & Range & - & $(5.5 \%, 5.4 \%)$ & $(4.9 \%, 5.0 \%)$ & $(4.7 \%, 4.8 \%)$ & $(2.1 \%, 3.1 \%)$ & $(3.7 \%, 7.1 \%)$ \\
\hline \multirow{6}{*}{$\begin{array}{l}\text { Smoke-free } \\
\text { air law alone }\end{array}$} & CIG & $34.2 \%$ & $25.8 \%$ & $22.2 \%$ & $21.5 \%$ & $-2.8 \%$ & $-2.9 \%$ \\
\hline & Range & - & $(26.1 \%, 25.4 \%)$ & $(22.5 \%, 21.9 \%)$ & $(21.9 \%, 21.2 \%)$ & $(-1.4 \%,-4.2 \%)$ & $(-1.4 \%,-4.3 \%)$ \\
\hline & Dual & $2.1 \%$ & $1.9 \%$ & $1.7 \%$ & $1.6 \%$ & $-0.4 \%$ & $-0.3 \%$ \\
\hline & Range & - & $(1.9 \%, 1.9 \%)$ & $(1.7 \%, 1.7 \%)$ & $(1.6 \%, 1.6 \%)$ & $(-0.2 \%,-0.6 \%)$ & $(-0.1 \%,-0.4 \%)$ \\
\hline & SLT & $7.1 \%$ & $5.6 \%$ & $4.8 \%$ & $4.5 \%$ & $0.2 \%$ & $0.3 \%$ \\
\hline & Range & - & $(5.6 \%, 5.6 \%)$ & $(4.8 \%, 4.8 \%)$ & $(4.5 \%, 4.5 \%)$ & $(0.1 \%, 0.2 \%)$ & $(0.1 \%, 0.4 \%)$ \\
\hline \multirow{6}{*}{$\begin{array}{l}\text { Media } \\
\text { campaign } \\
\text { alone }\end{array}$} & $\mathrm{CIG}$ & $34.2 \%$ & $26.3 \%$ & $22.8 \%$ & $22.2 \%$ & $-0.1 \%$ & $0.0 \%$ \\
\hline & Range & - & $(26.4 \%, 26.3 \%)$ & $(22.8 \%, 22.8 \%)$ & $(22.2 \%, 22.2 \%)$ & $(-0.1 \%,-0.2 \%)$ & $(0.0 \%, 0.0 \%)$ \\
\hline & Dual & $2.1 \%$ & $1.9 \%$ & $1.7 \%$ & $1.6 \%$ & $-0.1 \%$ & $0.0 \%$ \\
\hline & Range & - & $(1.9 \%, 1.9 \%)$ & $(1.7 \%, 1.7 \%)$ & $(1.6 \%, 1.6 \%)$ & $(-0.1 \%,-0.2 \%)$ & $(0.0 \%, 0.0 \%)$ \\
\hline & SLT & $7.1 \%$ & $5.6 \%$ & $4.8 \%$ & $4.5 \%$ & $-0.1 \%$ & $0.0 \%$ \\
\hline & Range & - & $(5.6 \%, 5.5 \%)$ & $(4.8 \%, 4.8 \%)$ & $(4.5 \%, 4.5 \%)$ & $(0.0 \%,-0.2 \%)$ & $(0.0 \%, 0.0 \%)$ \\
\hline \multirow{6}{*}{$\begin{array}{l}\text { Cessation } \\
\text { treatment } \\
\text { alone }\end{array}$} & $\mathrm{CIG}$ & $34.2 \%$ & $25.8 \%$ & $22.3 \%$ & $21.7 \%$ & $-2.4 \%$ & $-2.1 \%$ \\
\hline & Range & - & $(26.1 \%, 25.5 \%)$ & $(22.6 \%, 22.1 \%)$ & $(21.9 \%, 21.5 \%)$ & $(-1.2 \%,-3.5 \%)$ & $(-1.1 \%,-3.2 \%)$ \\
\hline & Dual & $2.1 \%$ & $1.8 \%$ & $1.7 \%$ & $1.6 \%$ & $-1.9 \%$ & $-1.6 \%$ \\
\hline & Range & - & $(1.9 \%, 1.8 \%)$ & $(1.7 \%, 1.7 \%)$ & $(1.6 \%, 1.6 \%)$ & $(-0.9 \%,-2.7 \%)$ & $(-0.8 \%,-2.4 \%)$ \\
\hline & SLT & $7.1 \%$ & $5.5 \%$ & $4.7 \%$ & $4.4 \%$ & $-1.5 \%$ & $-1.3 \%$ \\
\hline & Range & - & $(5.5 \%, 5.4 \%)$ & $(4.8 \%, 4.7 \%)$ & $(4.5 \%, 4.4 \%)$ & $(-0.4 \%,-2.5 \%)$ & $(-0.4 \%,-2.2 \%)$ \\
\hline \multirow{6}{*}{$\begin{array}{l}\text { Health } \\
\text { warning } \\
\text { alone }\end{array}$} & $\mathrm{CIG}$ & $34.2 \%$ & $26.4 \%$ & $22.9 \%$ & $22.2 \%$ & $0.0 \%$ & $0.0 \%$ \\
\hline & Range & - & $(26.4 \%, 26.4 \%)$ & $(22.9 \%, 22.9 \%)$ & $(22.2 \%, 22.2 \%)$ & $(0.0 \%, 0.0 \%)$ & $(0.0 \%, 0.0 \%)$ \\
\hline & Dual & $2.1 \%$ & $1.9 \%$ & $1.7 \%$ & $1.6 \%$ & $0.0 \%$ & $0.0 \%$ \\
\hline & Range & - & $(1.9 \%, 1.9 \%)$ & $(1.7 \%, 1.7 \%)$ & $(1.6 \%, 1.6 \%)$ & $(0.0 \%, 0.0 \%)$ & $(0.0 \%, 0.1 \%)$ \\
\hline & SLT & $7.1 \%$ & $5.5 \%$ & $4.7 \%$ & $4.4 \%$ & $-1.5 \%$ & $-1.6 \%$ \\
\hline & Range & - & $(5.5 \%, 5.5 \%)$ & $(4.8 \%, 4.7 \%)$ & $(4.5 \%, 4.4 \%)$ & $(-0.8 \%,-2.3 \%)$ & $(-0.8 \%,-2.3 \%)$ \\
\hline
\end{tabular}




\begin{tabular}{|c|c|c|c|c|c|c|c|}
\hline Scenario & & 1993 & 2018 & 2040 & 2060 & $\begin{array}{c}\text { Relative Difference } \\
\text { Compared to } \\
\text { Counterfactual in } 2040\end{array}$ & $\begin{array}{c}\text { Relative Difference } \\
\text { Compared to Counterfactual } \\
\text { in } 2060\end{array}$ \\
\hline \multirow{5}{*}{$\begin{array}{l}\text { Youth access } \\
\text { alone }\end{array}$} & $\mathrm{CIG}$ & $34.2 \%$ & $25.7 \%$ & $21.6 \%$ & $20.7 \%$ & $-5.6 \%$ & $-6.8 \%$ \\
\hline & Range & - & $(26.1 \%, 25.2 \%)$ & $(22.3 \%, 20.8 \%)$ & $(21.5 \%, 19.7 \%)$ & $(-2.6 \%,-9.1 \%)$ & $(-3.2 \%,-11.1 \%)$ \\
\hline & Dual & $2.1 \%$ & $1.9 \%$ & $1.7 \%$ & $1.6 \%$ & $-2.8 \%$ & $-3.5 \%$ \\
\hline & Range & - & $(1.9 \%, 1.8 \%)$ & $(1.7 \%, 1.6 \%)$ & $(1.6 \%, 1.5 \%)$ & $(-1.3 \%,-4.6 \%)$ & $(-1.6 \%,-5.8 \%)$ \\
\hline & Range & $34.2 \%$ & $(5.6 \%, 5.5 \%)$ & $(4.8 \%, 4.7 \%)$ & $(4.5 \%, 4.4 \%)$ & $(0.4 \%,-1.8 \%)$ & $(0.4 \%,-2.5 \%)$ \\
\hline \multicolumn{8}{|c|}{ Female } \\
\hline \multirow{5}{*}{$\begin{array}{l}\text { Counter- } \\
\text { factual }\end{array}$} & CIG & $29.5 \%$ & $26.5 \%$ & $23.7 \%$ & $23.2 \%$ & - & - \\
\hline & Range & - & $(26.5 \%, 26.5 \%)$ & $(23.7 \%, 23.7 \%)$ & $(23.2 \%, 23.2 \%)$ & - & - \\
\hline & Dual & $0.0 \%$ & $0.0 \%$ & $0.0 \%$ & $0.0 \%$ & - & - \\
\hline & Range & - & $(0.0 \%, 0.0 \%)$ & $(0.0 \%, 0.0 \%)$ & $(0.0 \%, 0.0 \%)$ & - & - \\
\hline & Range & - & $(0.1 \%, 0.1 \%)$ & $(0.1 \%, 0.1 \%)$ & $(0.1 \%, 0.1 \%)$ & - & - \\
\hline \multirow{6}{*}{ Status quo } & $\mathrm{CIG}$ & $29.5 \%$ & $20.4 \%$ & $16.6 \%$ & $15.6 \%$ & $-30.1 \%$ & $-32.6 \%$ \\
\hline & Range & - & $(22.2 \%, 18.7 \%)$ & $(18.7 \%, 14.6 \%)$ & $(17.8 \%, 13.6 \%)$ & $(-21.3 \%,-38.4 \%)$ & $(-23.1 \%,-41.4 \%)$ \\
\hline & Dual & $0.0 \%$ & $0.0 \%$ & $0.0 \%$ & $0.0 \%$ & - & - \\
\hline & Range & - & $(0.0 \%, 0.0 \%)$ & $(0.0 \%, 0.0 \%)$ & $(0.0 \%, 0.0 \%)$ & - & - \\
\hline & SLT & $0.5 \%$ & $0.1 \%$ & $0.1 \%$ & $0.1 \%$ & $6.2 \%$ & $12.1 \%$ \\
\hline & Range & - & $(0.1 \%, 0.1 \%)$ & $(0.1 \%, 0.1 \%)$ & $(0.1 \%, 0.1 \%)$ & $(5.6 \%, 6.7 \%)$ & $(9.5 \%, 14.5 \%)$ \\
\hline \multirow{6}{*}{ Price alone } & $\mathrm{CIG}$ & $29.5 \%$ & $21.9 \%$ & $18.4 \%$ & $17.5 \%$ & $-22.5 \%$ & $-24.4 \%$ \\
\hline & Range & - & $(22.9 \%, 20.8 \%)$ & $(19.6 \%, 17.2 \%)$ & $(18.8 \%, 16.3 \%)$ & $(-17.4 \%,-27.4 \%)$ & $(-18.9 \%,-29.6 \%)$ \\
\hline & Dual & $0.0 \%$ & $0.0 \%$ & $0.0 \%$ & $0.0 \%$ & - & - \\
\hline & Range & - & $(0.0 \%, 0.0 \%)$ & $(0.0 \%, 0.0 \%)$ & $(0.0 \%, 0.0 \%)$ & - & - \\
\hline & SLT & $0.5 \%$ & $0.1 \%$ & $0.1 \%$ & $0.1 \%$ & $7.4 \%$ & $12.5 \%$ \\
\hline & Range & - & $(0.1 \%, 0.1 \%)$ & $(0.1 \%, 0.1 \%)$ & $(0.1 \%, 0.1 \%)$ & $(5.8 \%, 8.9 \%)$ & $(9.3 \%, 15.6 \%)$ \\
\hline \multirow{4}{*}{$\begin{array}{l}\text { Smoke-free } \\
\text { air law alone }\end{array}$} & CIG & $29.5 \%$ & $25.8 \%$ & $23.1 \%$ & $22.6 \%$ & $-2.7 \%$ & $-2.7 \%$ \\
\hline & Range & - & $(0.0 \%, 0.0 \%)$ & $(0.0 \%, 0.0 \%)$ & $(0.0 \%, 0.0 \%)$ & - & - \\
\hline & SLT & $0.5 \%$ & $0.1 \%$ & $0.1 \%$ & $0.1 \%$ & $0.6 \%$ & $1.0 \%$ \\
\hline & Range & - & $(0.1 \%, 0.1 \%)$ & $(0.1 \%, 0.1 \%)$ & $(0.1 \%, 0.1 \%)$ & $(0.3 \%, 0.9 \%)$ & $(0.5 \%, 1.5 \%)$ \\
\hline \multirow{6}{*}{$\begin{array}{l}\text { Media } \\
\text { campaign } \\
\text { alone }\end{array}$} & CIG & $29.5 \%$ & $26.4 \%$ & $23.7 \%$ & $23.2 \%$ & $-0.2 \%$ & $0.0 \%$ \\
\hline & Range & - & $(26.4 \%, 26.3 \%)$ & $(23.7 \%, 23.7 \%)$ & $(23.2 \%, 23.2 \%)$ & $(-0.1 \%,-0.2 \%)$ & $(0.0 \%, 0.0 \%)$ \\
\hline & Dual & $0.0 \%$ & $0.0 \%$ & $0.0 \%$ & $0.0 \%$ & - & - \\
\hline & Range & - & $(0.0 \%, 0.0 \%)$ & $(0.0 \%, 0.0 \%)$ & $(0.0 \%, 0.0 \%)$ & - & - \\
\hline & SLT & $0.5 \%$ & $0.1 \%$ & $0.1 \%$ & $0.1 \%$ & $-0.1 \%$ & $0.0 \%$ \\
\hline & Range & - & $(0.1 \%, 0.1 \%)$ & $(0.1 \%, 0.1 \%)$ & $(0.1 \%, 0.1 \%)$ & $(0.0 \%,-0.1 \%)$ & $(0.0 \%, 0.0 \%)$ \\
\hline \multirow{6}{*}{$\begin{array}{l}\text { Cessation } \\
\text { treatment } \\
\text { alone }\end{array}$} & CIG & $29.5 \%$ & $25.9 \%$ & $23.2 \%$ & $22.8 \%$ & $-2.1 \%$ & $-1.8 \%$ \\
\hline & Range & - & $(26.2 \%, 25.6 \%)$ & $(23.5 \%, 23.0 \%)$ & $(23.0 \%, 22.6 \%)$ & $(-1.1 \%,-3.1 \%)$ & $(-0.9 \%,-2.6 \%)$ \\
\hline & Dual & $0.0 \%$ & $0.0 \%$ & $0.0 \%$ & $0.0 \%$ & - & - \\
\hline & Range & - & $(0.0 \%, 0.0 \%)$ & $(0.0 \%, 0.0 \%)$ & $(0.0 \%, 0.0 \%)$ & - & - \\
\hline & SLT & $0.5 \%$ & $0.1 \%$ & $0.1 \%$ & $0.1 \%$ & $-1.6 \%$ & $-1.4 \%$ \\
\hline & Range & - & $(0.1 \%, 0.1 \%)$ & $(0.1 \%, 0.1 \%)$ & $(0.1 \%, 0.1 \%)$ & $(-0.4 \%,-2.7 \%)$ & $(-0.4 \%,-2.5 \%)$ \\
\hline \multirow{6}{*}{$\begin{array}{l}\text { Health } \\
\text { warning } \\
\text { alone }\end{array}$} & $\mathrm{CIG}$ & $29.5 \%$ & $26.5 \%$ & $23.7 \%$ & $23.2 \%$ & $0.0 \%$ & $0.0 \%$ \\
\hline & Range & - & $(26.5 \%, 26.5 \%)$ & $(23.7 \%, 23.7 \%)$ & $(23.2 \%, 23.2 \%)$ & $(0.0 \%, 0.0 \%)$ & $(0.0 \%, 0.0 \%)$ \\
\hline & Dual & $0.0 \%$ & $0.0 \%$ & $0.0 \%$ & $0.0 \%$ & - & - \\
\hline & Range & - & $(0.0 \%, 0.0 \%)$ & $(0.0 \%, 0.0 \%)$ & $(0.0 \%, 0.0 \%)$ & - & - \\
\hline & SLT & $0.5 \%$ & $0.1 \%$ & $0.1 \%$ & $0.1 \%$ & $-1.7 \%$ & $-1.7 \%$ \\
\hline & Range & - & $(0.1 \%, 0.1 \%)$ & $(0.1 \%, 0.1 \%)$ & $(0.1 \%, 0.1 \%)$ & $(-0.9 \%,-2.6 \%)$ & $(-0.9 \%,-2.6 \%)$ \\
\hline \multirow{6}{*}{$\begin{array}{l}\text { Youth access } \\
\text { alone }\end{array}$} & CIG & $29.5 \%$ & $25.9 \%$ & $22.6 \%$ & $21.7 \%$ & $-5.0 \%$ & $-6.4 \%$ \\
\hline & Range & - & $(26.2 \%, 25.5 \%)$ & $(23.2 \%, 21.8 \%)$ & $(22.5 \%, 20.8 \%)$ & $(-2.3 \%,-8.0 \%)$ & $(-3.0 \%,-10.3 \%)$ \\
\hline & Dual & $0.0 \%$ & $0.0 \%$ & $0.0 \%$ & $0.0 \%$ & - & - \\
\hline & Range & - & $(0.0 \%, 0.0 \%)$ & $(0.0 \%, 0.0 \%)$ & $(0.0 \%, 0.0 \%)$ & - & - \\
\hline & SLT & $0.5 \%$ & $0.1 \%$ & $0.1 \%$ & $0.1 \%$ & $2.0 \%$ & $3.0 \%$ \\
\hline & Range & - & $(0.1 \%, 0.1 \%)$ & $(0.1 \%, 0.1 \%)$ & $(0.1 \%, 0.1 \%)$ & $(0.9 \%, 3.2 \%)$ & $(1.4 \%, 4.9 \%)$ \\
\hline
\end{tabular}

Abbreviations: CIG, cigarette; SLT, smokeless tobacco; Dual, dual use of cigarette and smokeless tobacco.

*The reported prevalence is the best estimate with its left/right bound range from model predictions using lower/upper policy effect sizes.

** Policies fixed at 1993 level. 
Table 4. Tobacco-Attributable Deaths and Deaths averted* for Both Genders in Each Individual Policy Scenario, 1993-2060

\begin{tabular}{|c|c|c|c|c|c|c|c|}
\hline \multicolumn{8}{|c|}{ Male } \\
\hline $\begin{array}{l}\text { Tobacco-attrib } \\
\text { deaths }\end{array}$ & utable & 1993 & 2018 & 2040 & 2060 & $\begin{array}{l}\text { Cumulative } \\
\text { Through } 2040\end{array}$ & $\begin{array}{l}\text { Cumulative } \\
\text { Through } 2060\end{array}$ \\
\hline \multirow{8}{*}{ Status quo } & CIG & 4554 & 5359 & 4503 & 3607 & 245372 & 324358 \\
\hline & Range & - & $(5546,5181)$ & $(4830,4199)$ & $(4039,3210)$ & $(252$ 977, 238 192) & (339 558, 310 176) \\
\hline & Dual & 352 & 240 & 348 & 296 & 13476 & 19942 \\
\hline & Range & - & $(247,234)$ & $(366,331)$ & $(317,275)$ & $(13818,13$ 147) & $(20682,19225)$ \\
\hline & SLT & 189 & 167 & 201 & 177 & 8839 & 12661 \\
\hline & Range & - & $(172,163)$ & $(209,193)$ & $(184,169)$ & $(9033,8653)$ & $(13020,12314)$ \\
\hline & Total & 5096 & 5767 & 5052 & 4080 & 267688 & 356960 \\
\hline & Range & - & $(5964,5578)$ & $(5405,4723)$ & $(4541,3655)$ & $(275828,259992)$ & (373 260, 341715$)$ \\
\hline \multirow{7}{*}{$\begin{array}{l}\text { Counter- } \\
\text { factual }\end{array}$} & $\mathrm{CIG}$ & 4554 & 5974 & 5601 & 5082 & 270599 & 375500 \\
\hline & Range & - & $(5974,5974)$ & $(5601,5601)$ & $(5082,5082)$ & $(270599,270599)$ & $(375$ 500, 375 500) \\
\hline & Range & - & $(263,263)$ & $(408,408)$ & $(366,366)$ & $(14$ 637, 14 637) & $(22441,22441)$ \\
\hline & SLT & 189 & 176 & 215 & 187 & 9199 & 13287 \\
\hline & Range & - & $(176,176)$ & $(215,215)$ & $(187,187)$ & $(9199,9199)$ & $(13287,13287)$ \\
\hline & Total & 5096 & 6413 & 6225 & 5636 & 294434 & 411228 \\
\hline & Range & - & $(6413,6413)$ & $(6225,6225)$ & $(5636,5636)$ & $(294434,294$ 434) & (411 228, 411228$)$ \\
\hline Deaths averte & & 1993 & 2018 & 2040 & 2060 & $\begin{array}{l}\text { Cumulative through } \\
\qquad 2040\end{array}$ & Cumulative Through 2060 \\
\hline \multirow{6}{*}{ Status quo } & $\mathrm{CIG}$ & 0 & 615 & 1098 & 1475 & 25227 & 51142 \\
\hline & Range & - & $(428,793)$ & $(771,1402)$ & $(1043,1872)$ & $(17622,32$ 407) & (35 941, 65 323) \\
\hline & Dual & 0 & 23 & 60 & 70 & 1160 & 2499 \\
\hline & Range & - & $(16,29)$ & $(42,77)$ & $(49,91)$ & $(818,1489)$ & $(1759,3216)$ \\
\hline & SLT & 0 & 9 & 14 & 11 & 359 & 626 \\
\hline & Range & - & $(448,835)$ & $(819,1501)$ & $(1095,1981)$ & $(18606,34442)$ & $(37967,69512)$ \\
\hline \multirow{8}{*}{ Price alone } & $\mathrm{CIG}$ & 0 & 455 & 798 & 1081 & 18545 & 37587 \\
\hline & Range & - & $(348,560)$ & $(615,970)$ & $(836,1311)$ & $(14$ 214, 22 697) & $(28933,45812)$ \\
\hline & Dual & 0 & 18 & 46 & 53 & 910 & 1938 \\
\hline & Range & - & $(14,22)$ & $(35,57)$ & $(40,65)$ & $(694,1119)$ & $(1478,2382)$ \\
\hline & SLT & 0 & 7 & 8 & 2 & 257 & 375 \\
\hline & Range & - & $(4,10)$ & $(4,12)$ & $(1,4)$ & $(130,381)$ & $(185,561)$ \\
\hline & Total & 0 & 481 & 852 & 1136 & 19712 & 39900 \\
\hline & Range & - & $(365,592)$ & $(654,1039)$ & $(877,1381)$ & $(15$ 038, 24 197) & $(30596,48755)$ \\
\hline \multirow{8}{*}{$\begin{array}{l}\text { Smoke free } \\
\text { air law alone }\end{array}$} & CIG & 0 & 72 & 129 & 137 & 2940 & 5586 \\
\hline & Range & - & $(36,108)$ & $(65,192)$ & $(69,204)$ & $(1478,4386)$ & $(2809,8330)$ \\
\hline & Dual & 0 & 0 & 0 & 0 & 0 & -1 \\
\hline & Range & - & $(0,0)$ & $(0,0)$ & $(0,0)$ & $(0,0)$ & $(0,-1)$ \\
\hline & SLT & 0 & 1 & 2 & 1 & 45 & 83 \\
\hline & Range & - & $(0,1)$ & $(1,3)$ & $(1,2)$ & $(22,67)$ & $(41,124)$ \\
\hline & Total & 0 & 73 & 131 & 138 & 2984 & 5668 \\
\hline & Range & - & $(37,109)$ & $(66,196)$ & $(70,206)$ & $(1500,4453)$ & $(2850,8454)$ \\
\hline \multirow{6}{*}{$\begin{array}{l}\text { Media } \\
\text { campaign } \\
\text { alone }\end{array}$} & $\mathrm{CIG}$ & 0 & 34 & 21 & 3 & 976 & 1200 \\
\hline & Range & - & $(17,51)$ & $(10,31)$ & $(2,5)$ & $(488,1463)$ & $(600,1800)$ \\
\hline & Dual & 0 & 2 & 2 & 0 & 54 & 72 \\
\hline & Range & - & $(1,2)$ & $(1,2)$ & $(0,0)$ & $(27,81)$ & $(36,108)$ \\
\hline & SLT & 0 & 0 & 1 & 0 & 17 & 25 \\
\hline & Range & - & $(0,1)$ & $(0,1)$ & $(0,0)$ & $(4,29)$ & $(6,44)$ \\
\hline
\end{tabular}




\begin{tabular}{|c|c|c|c|c|c|c|c|}
\hline \multicolumn{8}{|c|}{ Male } \\
\hline \multicolumn{2}{|c|}{$\begin{array}{l}\text { Tobacco-attributable } \\
\text { deaths }\end{array}$} & 1993 & 2018 & 2040 & 2060 & $\begin{array}{c}\text { Cumulative } \\
\text { Through } 2040 \\
\end{array}$ & $\begin{array}{c}\text { Cumulative } \\
\text { Through } 2060 \\
\end{array}$ \\
\hline \multirow{8}{*}{$\begin{array}{l}\text { Cessation } \\
\text { treatment } \\
\text { alone }\end{array}$} & CIG & 0 & 89 & 161 & 135 & 3840 & 6805 \\
\hline & Range & - & $(45,132)$ & $(82,238)$ & $(69,199)$ & $(1940,5703)$ & $(3446,10083)$ \\
\hline & Dual & 0 & 3 & 10 & 7 & 173 & 349 \\
\hline & Range & - & $(1,4)$ & $(5,14)$ & $(4,11)$ & $(87,258)$ & $(176,518)$ \\
\hline & SLT & 0 & 1 & 4 & 4 & 70 & 151 \\
\hline & Range & - & $(0,2)$ & $(1,7)$ & $(1,7)$ & $(18,122)$ & $(38,261)$ \\
\hline & Total & 0 & 93 & 174 & 146 & 4084 & 7305 \\
\hline & Range & - & $(47,139)$ & $(88,259)$ & $(73,217)$ & $(2045,6082)$ & $(3660,10862)$ \\
\hline \multirow{8}{*}{$\begin{array}{l}\text { Health } \\
\text { warning alone }\end{array}$} & $\mathrm{CIG}$ & - & - & - & - & - & - \\
\hline & Range & & & & & & \\
\hline & Dual & - & - & - & - & - & - \\
\hline & Range & & & & & & \\
\hline & SLT & 0 & 1 & 3 & 3 & 48 & 111 \\
\hline & Range & - & $(0,1)$ & $(1,4)$ & $(2,5)$ & $(24,72)$ & $(56,166)$ \\
\hline & Total & 0 & 1 & 3 & 2 & 46 & 95 \\
\hline & Range & - & $(0,1)$ & $(1,4)$ & $(1,3)$ & $(23,69)$ & $(48,141)$ \\
\hline \multirow{8}{*}{$\begin{array}{l}\text { Youth access } \\
\text { alone }\end{array}$} & $\mathrm{CIG}$ & 0 & 4 & 82 & 250 & 809 & 4,111 \\
\hline & Range & - & $(2,5)$ & $(39,130)$ & $(118,400)$ & $(387,1276)$ & $(1952,6538)$ \\
\hline & Dual & 0 & 0 & 3 & 10 & 33 & 163 \\
\hline & Range & - & $(0,0)$ & $(2,5)$ & $(5,16)$ & $(16,53)$ & $(77,264)$ \\
\hline & SLT & 0 & 0 & 0 & 1 & 3 & 17 \\
\hline & Range & - & $(0,0)$ & $(0,1)$ & $(0,3)$ & $(0,7)$ & $(0,39)$ \\
\hline & Total & 0 & 4 & 86 & 261 & 845 & 4,292 \\
\hline & Range & - & $(2,6)$ & $(41,136)$ & $(122,418)$ & $(403,1336)$ & $(2026,6841)$ \\
\hline \multicolumn{8}{|c|}{ Female } \\
\hline $\begin{array}{l}\text { Tobacco-Attribu } \\
\text { Deaths }\end{array}$ & utable & 1993 & 2018 & 2040 & 2060 & $\begin{array}{c}\text { Cumulative } \\
\text { Through } 2040 \\
\end{array}$ & $\begin{array}{c}\text { Cumulative } \\
\text { Through } 2060 \\
\end{array}$ \\
\hline \multirow{6}{*}{ Status quo } & $\mathrm{CIG}$ & 2313 & 3347 & 3343 & 2531 & 150680 & 208390 \\
\hline & Range & - & $(3464,3235)$ & $(3576,3126)$ & $(2842,2246)$ & (155 714, 145 918) & $(218892,198608)$ \\
\hline & SLT & 31 & 10 & 8 & 6 & 777 & 905 \\
\hline & Range & - & $(10,10)$ & $(8,8)$ & $(6,6)$ & $(791,764)$ & $(921,889)$ \\
\hline & Total & 2344 & 3357 & 3351 & 2537 & 151457 & 209295 \\
\hline & Range & - & $(3474,3245)$ & $(3584,3133)$ & $(2848,2252)$ & $(156505,146682)$ & $(219813,199$ 497) \\
\hline \multirow{5}{*}{$\begin{array}{l}\text { Counter- } \\
\text { factual }\end{array}$} & $\mathrm{CIG}$ & 2313 & 3732 & 4116 & 3613 & 167225 & 243642 \\
\hline & Range & - & $(3732,3732)$ & $(4116,4116)$ & $(3613,3613)$ & $(167225,167225)$ & $(243642,243642)$ \\
\hline & SLT & 31 & 11 & 8 & 6 & 803 & 932 \\
\hline & Range & - & $(11,11)$ & $(8,8)$ & $(6,6)$ & $(803,803)$ & $(932,932)$ \\
\hline & Total & 2344 & 3742 & 4125 & 3619 & 168028 & 244574 \\
\hline \multicolumn{2}{|l|}{ Deaths Averted } & 1993 & 2018 & 2040 & 2060 & $\begin{array}{c}\text { Cumulative } \\
\text { Through } 2040\end{array}$ & $\begin{array}{c}\text { Cumulative } \\
\text { Through } 2060\end{array}$ \\
\hline \multirow{6}{*}{ Status quo } & $\mathrm{CIG}$ & 0 & 385 & 774 & 1082 & 16546 & 35252 \\
\hline & Range & - & $(268,497)$ & $(540,991)$ & $(771,1367)$ & $(11511,21$ 307) & $(24750,45034)$ \\
\hline & SLT & 0 & 1 & 0 & 0 & 26 & 28 \\
\hline & Range & - & $(0,1)$ & $(0,1)$ & $(0,0)$ & $(12,39)$ & $(11,43)$ \\
\hline & Total & 0 & 385 & 774 & 1082 & 16571 & 35279 \\
\hline & Range & - & $(268,498)$ & $(540,992)$ & $(770,1367)$ & $(11523,21346)$ & $(24761,45$ 077) \\
\hline \multirow{6}{*}{ Price alone } & CIG & 0 & 285 & 557 & 809 & 12064 & 25907 \\
\hline & Range & - & $(217,351)$ & $(429,678)$ & $(627,979)$ & $(9234,14780)$ & $(19937,31575)$ \\
\hline & SLT & 0 & 1 & 0 & 0 & 20 & 19 \\
\hline & Range & - & $(0,1)$ & $(0,0)$ & $(0,0)$ & $(10,30)$ & $(8,30)$ \\
\hline & Total & 0 & 286 & 557 & 809 & 12084 & 25926 \\
\hline & Range & - & $(218,352)$ & $(429,679)$ & $(627,979)$ & $(9245,14811)$ & (19 946, 31 604) \\
\hline
\end{tabular}




\begin{tabular}{|c|c|c|c|c|c|c|c|}
\hline \multicolumn{8}{|c|}{ Female } \\
\hline Deaths Avertec & & 1993 & 2018 & 2040 & 2060 & $\begin{array}{l}\text { Cumulative } \\
\text { Through } 2040\end{array}$ & $\begin{array}{l}\text { Cumulative } \\
\text { Through } 2060\end{array}$ \\
\hline \multirow{6}{*}{$\begin{array}{l}\text { Smoke free } \\
\text { air law alone }\end{array}$} & $\mathrm{ClG}$ & 0 & 45 & 95 & 95 & 2021 & 3931 \\
\hline & Range & - & $(23,67)$ & $(48,142)$ & $(48,142)$ & $(1016,3015)$ & $(1977,5861)$ \\
\hline & SLT & 0 & 0 & 0 & 0 & 0 & 0 \\
\hline & Range & - & $(0,0)$ & $(0,0)$ & $(0,0)$ & $(0,0)$ & $(0,0)$ \\
\hline & Total & 0 & 45 & 95 & 95 & 2021 & 3930 \\
\hline & Range & - & $(23,67)$ & $(48,142)$ & $(48,142)$ & $(1016,3015)$ & $(1977,5861)$ \\
\hline \multirow{6}{*}{$\begin{array}{l}\text { Media camp- } \\
\text { aign alone }\end{array}$} & $\mathrm{CIG}$ & 0 & 23 & 20 & 5 & 706 & 962 \\
\hline & Range & - & $(11,34)$ & $(10,30)$ & $(3,8)$ & $(353,1059)$ & $(481,1443)$ \\
\hline & SLT & 0 & 0 & 0 & 0 & 1 & 2 \\
\hline & Range & - & $(0,0)$ & $(0,0)$ & $(0,0)$ & $(0,2)$ & $(0,3)$ \\
\hline & Total & 0 & 23 & 20 & 5 & 708 & 963 \\
\hline & Range & - & $(11,34)$ & $(10,30)$ & $(3,8)$ & $(354,1062)$ & $(481,1446)$ \\
\hline \multirow{6}{*}{$\begin{array}{l}\text { Cessation } \\
\text { treatment } \\
\text { alone }\end{array}$} & $\mathrm{CIG}$ & 0 & 56 & 130 & 109 & 2706 & 5153 \\
\hline & Range & - & $(28,83)$ & $(66,193)$ & $(55,161)$ & $(1367,4020)$ & $(2608,7635)$ \\
\hline & SLT & 0 & 0 & 0 & 0 & 4 & 6 \\
\hline & Range & - & $(0,0)$ & $(0,0)$ & $(0,0)$ & $(1,7)$ & $(2,11)$ \\
\hline & Total & 0 & 56 & 130 & 109 & 2711 & 5159 \\
\hline & Range & - & $(28,83)$ & $(66,193)$ & $(55,161)$ & $(1368,4027)$ & $(2610,7646)$ \\
\hline \multirow{6}{*}{$\begin{array}{l}\text { Health } \\
\text { warning alone }\end{array}$} & CIG & - & - & - & - & - & - \\
\hline & Range & & & & & & \\
\hline & SLT & 0 & 0 & 0 & 0 & 3 & 5 \\
\hline & Range & - & $(0,0)$ & $(0,0)$ & $(0,0)$ & $(1,4)$ & $(2,7)$ \\
\hline & Total & 0 & 0 & 0 & 0 & 3 & 5 \\
\hline & Range & - & $(0,0)$ & $(0,0)$ & $(0,0)$ & $(1,4)$ & $(2,7)$ \\
\hline \multirow{6}{*}{$\begin{array}{l}\text { Youth access } \\
\text { alone }\end{array}$} & $\mathrm{CIG}$ & 0 & 0 & 0 & 0 & 0 & 0 \\
\hline & Range & - & $(0,0)$ & $(0,0)$ & $(0,0)$ & $(0,0)$ & $(0,0)$ \\
\hline & SLT & 0 & 0 & 0 & 0 & 0 & 0 \\
\hline & Range & - & $(0,0)$ & $(0,0)$ & $(0,0)$ & $(0,0)$ & $(0,0)$ \\
\hline & Total & 0 & 0 & 0 & 0 & 0 & 0 \\
\hline & Range & - & $(0,0)$ & $(0,0)$ & $(0,0)$ & $(0,0)$ & $(0,0)$ \\
\hline
\end{tabular}

Abbreviations: CIG, cigarette; SLT, smokeless tobacco; Dual, dual use of cigarette and smokeless tobacco.

*The reported deaths averted is given as the best estimate with its left/right bound range from model predictions using lower/upper policy effect sizes.

$(23.0 \%, 41.6 \%)$ for male and $32.6 \%(23.1 \%, 41.4 \%)$ for female exclusive smokers, $20.2 \%(14.0 \%, 26.6 \%)$ for male dual users, and $0.9 \%(-1.8 \%, 3.2 \%)$ for male and $12.1 \%(10.0 \%, 14.0 \%)$ for female exclusive SLT users. Compared to the counterfactual, SimSmoke estimates that $9018(6336,11601)$ total tobaccoattributable deaths are averted (64\% male deaths averted) by 2018, increasing to $89547(62704,114612)$ averted deaths by 2060 .

By individual policy, increased prices (largely due to cigarette taxes) reduce exclusive cigarette use rates by an average of $17.5 \%$ for males and females by 2018 and avert 65,826 total deaths by 2060 . Smoke-free air laws yield a $2.5 \%$ reduction in exclusive cigarette use by 2018 and avert 9598 deaths by 2060 . Cessation treatment policies show a relative reduction of 2.2\% in 2018, with 12464 averted deaths by 2060 . Youth access restrictions show a $2.6 \%$ reduction in exclusive cigarette use and avert 6530 deaths by 2060 .

SimSmoke estimated the highest reduction in 2018 exclusive smokers' prevalence for taxation (68\%), followed by youth access policy (11\%), smoke-free air laws (10\%), cessation treatments (9\%), and tobacco control campaigns (2\%). For male dual prevalence, impacts were higher for taxation (76\%), cessation treatment (10\%), and youth access enforcement (9\%) but generally lower for other policies. For SLT use, the order is taxes (44\%), cessation programs (24\%), health warnings (24\%), and youth access policy (5\%).

\section{The Effect of Stronger Future Policies}

Tables 5 and 6 show the projected smoking prevalence and exclusive SLT use from strengthening tobacco control policies. Increasing the excise tax per pack of cigarettes by $\$ 2.00$ and SLT by $\$ 2.00$ reduces the average male and female smoking prevalence by $9.6 \%$, reduces male and female SLT use by $10.3 \%$ and $9.6 \%$ initially increasing to $18.5 \%$ and $12.7 \%$ by 2060 , averting 11,072 deaths by 2060 . An SLT tax increase of $\$ 2.00$ yields a $10.3 \%(5.2 \%, 15.2 \%)$ and a $9.6 \%$ $(4.8 \%, 14.1 \%)$ immediate relative reduction for male and female exclusive SLT users, increasing to $18.5 \%$ and $12.7 \%$ by 2060 and averting 615 deaths. Increasing smoke-free air laws to their highest level, as shown in Table 1, reduces 
Table 5. Kentucky Prevalence* of Cigarette/SLT/Dual Use for Future Policy Change Scenarios, 2019-2060

\begin{tabular}{|c|c|c|c|c|}
\hline \multicolumn{5}{|c|}{ Males } \\
\hline Overall smoking prevalence & 2018 & 2019 & 2040 & 2060 \\
\hline Status quo policies & $22 \%(24 \%, 20 \%)$ & $21 \%(23 \%, 20 \%)$ & $17 \%(19 \%, 15 \%)$ & $16 \%(18 \%, 14 \%)$ \\
\hline \multicolumn{5}{|l|}{ \% Change in Smoking Prevalence From Status Quo } \\
\hline Increase tax by $\$ 2$ & - & $-5.1 \%(-3.9 \%,-6.3 \%)$ & $-7.8 \%(-6.0 \%,-9.6 \%)$ & $-9.2 \%(-7.1 \%,-11.4 \%)$ \\
\hline Comprehensive smoke-free air laws and enforcement & - & $-5.1 \%(-2.5 \%,-7.6 \%)$ & $-6.3 \%(-3.1 \%,-9.5 \%)$ & $-6.6 \%(-3.3 \%,-10.0 \%)$ \\
\hline Comprehensive marketing ban and enforcement & - & $-4.1 \%(-2.1 \%,-6.2 \%)$ & $-6.4 \%(-3.2 \%,-9.7 \%)$ & $-7.6 \%(-3.8 \%,-11.5 \%)$ \\
\hline High intensity tobacco control campaigns & - & $-2.8 \%(-1.4 \%,-4.2 \%)$ & $-3.6 \%(-1.8 \%,-5.4 \%)$ & $-3.7 \%(-1.8 \%,-5.6 \%)$ \\
\hline Strong health warnings & - & $-3.0 \%(-1.5 \%,-4.6 \%)$ & $-6.0 \%(-3.0 \%,-9.1 \%)$ & $-7.0 \%(-3.5 \%,-10.5 \%)$ \\
\hline Strong youth access enforcement & - & $0.0 \%(0.0 \%, 0.0 \%)$ & $-4.2 \%(-1.7 \%,-7.5 \%)$ & $-7.1 \%(-2.9 \%,-12.8 \%)$ \\
\hline Cessation treatment policies & - & $-2.0 \%(-1.0 \%,-3.0 \%)$ & $-3.7 \%(-1.7 \%,-5.1 \%)$ & $-3.4 \%(-1.6 \%,-4.6 \%)$ \\
\hline All above policies with $\$ 2$ tax increase & - & $-23.4 \%(-13.5 \%,-32.3 \%)$ & $-36.9 \%(-21.3 \%,-50.4 \%)$ & $-41.3 \%(-24.1 \%,-55.8 \%)$ \\
\hline Exclusive SLT Use Prevalence & 2018 & 2019 & 2040 & 2060 \\
\hline Status quo policies & $5.3 \%(5.5 \%, 5.1 \%)$ & $5.3 \%(5.5 \%, 5.1 \%)$ & $4.7 \%(4.9 \%, 4.6 \%)$ & $4.5 \%(4.6 \%, 4.4 \%)$ \\
\hline \multicolumn{5}{|l|}{ \% Change in SLT Use Prevalence from Status Quo } \\
\hline Increase tax by $\$ 2$ & - & $-10.3 \%(-5.2 \%,-15.2 \%)$ & $-15.6 \%(-8.0 \%,-22.6 \%)$ & $-18.5 \%(-9.6 \%,-26.6 \%)$ \\
\hline Comprehensive. Smoke free laws and enforcement & - & $-0.4 \%(-0.1 \%,-0.6 \%)$ & $-0.3 \%(0.0 \%,-0.6 \%)$ & $-0.2 \%(0.1 \%,-0.5 \%)$ \\
\hline Comprehensive marketing ban and enforcement & - & $-4.4 \%(-1.1 \%,-7.8 \%)$ & $-6.3 \%(-1.5 \%,-11.1 \%)$ & $-7.3 \%(-1.7 \%,-13.0 \%)$ \\
\hline High Intensity tobacco control campaigns & - & $-1.2 \%(-0.3 \%,-2.2 \%)$ & $-1.5 \%(-0.4 \%,-2.7 \%)$ & $-1.5 \%(-0.3 \%,-2.7 \%)$ \\
\hline Strong health warnings & - & $-2.0 \%(-0.5 \%,-3.5 \%)$ & $-4.0 \%(-0.9 \%,-7.2 \%)$ & $-4.8 \%(-1.1 \%,-8.6 \%)$ \\
\hline Strong youth access enforcement & - & $0.0 \%(0.0 \%, 0.0 \%)$ & $-0.8 \%(0.1 \%,-2.1 \%)$ & $-1.5 \%(0.2 \%,-3.8 \%)$ \\
\hline Cessation treatment policies & - & $-1.0 \%(-0.2 \%,-1.8 \%)$ & $-2.0 \%(-0.5 \%,-3.1 \%)$ & $-1.9 \%(-0.5 \%,-3.0 \%)$ \\
\hline All of the above policies with $\$ 2$ tax increase & - & $-18.6 \%(-7.4 \%,-28.6 \%)$ & $-28.7 \%(-11.1 \%,-43.6 \%)$ & $-32.9 \%(-12.8 \%,-49.3 \%)$ \\
\hline \multicolumn{5}{|c|}{ Females } \\
\hline Overall smoking prevalence & 2018 & 2019 & 2040 & 2060 \\
\hline Status Quo Policies & $20 \%(22 \%, 19 \%)$ & $20 \%(22 \%, 18 \%)$ & $17 \%(19 \%, 15 \%)$ & $16 \%(18 \%, 14 \%)$ \\
\hline \multicolumn{5}{|l|}{ \% Change in Smoking Prevalence from Status Quo } \\
\hline Increase tax by $\$ 2$ & - & $-5.1 \%(-3.9 \%,-6.3 \%)$ & $-8.1 \%(-6.1 \%,-10.0 \%)$ & $-10.0 \%(-7.5 \%,-12.5 \%)$ \\
\hline Comprehensive. Smoke free laws and enforcement & - & $-5.3 \%(-2.7 \%,-8.0 \%)$ & $-6.3 \%(-3.1 \%,-9.5 \%)$ & $-6.5 \%(-3.2 \%,-9.8 \%)$ \\
\hline Comprehensive marketing ban and enforcement & - & $-4.1 \%(-2.0 \%,-6.1 \%)$ & $-6.0 \%(-3.0 \%,-9.0 \%)$ & $-7.2 \%(-3.6 \%,-10.9 \%)$ \\
\hline High intensity tobacco control campaigns & - & $-2.8 \%(-1.4 \%,-4.2 \%)$ & $-3.5 \%(-1.7 \%,-5.2 \%)$ & $-3.5 \%(-1.7 \%,-5.2 \%)$ \\
\hline Strong health warnings & - & $-3.0 \%(-1.5 \%,-4.5 \%)$ & $-5.4 \%(-2.7 \%,-8.3 \%)$ & $-6.3 \%(-3.1 \%,-9.5 \%)$ \\
\hline Strong youth access enforcement & - & $0.0 \%(0.0 \%, 0.0 \%)$ & $-3.6 \%(-1.5 \%,-6.5 \%)$ & $-6.7 \%(-2.8 \%,-12.2 \%)$ \\
\hline Cessation treatment policies & - & $-2.0 \%(-1.0 \%,-3.0 \%)$ & $-3.4 \%(-1.6 \%,-4.8 \%)$ & $-2.9 \%(-1.4 \%,-3.9 \%)$ \\
\hline All of the above policies with $\$ 2$ tax increase & - & $-23.7 \%(-15.5 \%,-31.3 \%)$ & $-35.6 \%(-23.0 \%,-47.0 \%)$ & $-40.1 \%(-26.4 \%,-52.4 \%)$ \\
\hline Exclusive SLT Use Prevalence & 2018 & 2019 & 2040 & 2060 \\
\hline Status quo policies & $0.1 \%(0.1 \%, 0.1 \%)$ & $0.1 \%(0.1 \%, 0.1 \%)$ & $0.1 \%(0.1 \%, 0.1 \%)$ & $0.1 \%(0.1 \%, 0.1 \%)$ \\
\hline \multicolumn{5}{|l|}{ \% Change in SLT Use Prevalence From Status Quo } \\
\hline Increase tax by $\$ 2$ & - & $-9.6 \%(-4.8 \%,-14.1 \%)$ & $-12.0 \%(-6.1 \%,-17.5 \%)$ & $-12.7 \%(-6.5 \%,-18.6 \%)$ \\
\hline Comprehensive. Smoke free laws and enforcement & - & $-0.4 \%(-0.1 \%,-0.6 \%)$ & $0.1 \%(0.2 \%,-0.1 \%)$ & $0.8 \%(0.6 \%, 0.8 \%)$ \\
\hline Comprehensive marketing ban and enforcement & - & $-4.4 \%(-1.1 \%,-7.8 \%)$ & $-6.7 \%(-1.5 \%,-12.0 \%)$ & $-7.1 \%(-1.2 \%,-13.1 \%)$ \\
\hline High intensity tobacco control campaigns & - & $-1.2 \%(-0.3 \%,-2.2 \%)$ & $-1.4 \%(-0.3 \%,-2.6 \%)$ & $-1.2 \%(-0.1 \%,-2.3 \%)$ \\
\hline Strong health warnings & - & $-2.0 \%(-0.5 \%,-3.5 \%)$ & $-4.5 \%(-1.0 \%,-8.0 \%)$ & $-4.8 \%(-0.8 \%,-8.9 \%)$ \\
\hline Strong youth access enforcement & - & $0.0 \%(0.0 \%, 0.0 \%)$ & $0.4 \%(0.2 \%, 0.7 \%)$ & $1.7 \%(0.8 \%, 2.7 \%)$ \\
\hline Cessation treatment policies & - & $-1.0 \%(-0.2 \%,-1.8 \%)$ & $-2.1 \%(-0.5 \%,-3.3 \%)$ & $-2.1 \%(-0.5 \%,-3.3 \%)$ \\
\hline All of the above policies with $\$ 2$ tax increase & - & $-17.9 \%(-7.1 \%,-27.7 \%)$ & $-25.0 \%(-8.9 \%,-38.9 \%)$ & $-24.6 \%(-7.7 \%,-39.4 \%)$ \\
\hline
\end{tabular}

Abbreviations: SLT, smokeless tobacco.

* The reported prevalence is the best estimate with its left/right bound range from model predictions using lower/upper policy effect sizes. 
Table 6. Sensitivity Analysis for Lives Saved* (Both Genders) for Stronger Future Policy Scenarios in Kentucky, 2019-2060

\begin{tabular}{|c|c|c|c|c|}
\hline \multicolumn{5}{|c|}{ Deaths Averted: All Smokers } \\
\hline Scenario & 2040 & 2060 & Cumulative** Through 2040 & Cumulative** Through 2060 \\
\hline Increase tax by $\$ 2$ & $284(237,318)$ & $404(350,435)$ & $3815(3143,4336)$ & $10457(8813,11604)$ \\
\hline $\begin{array}{l}\text { Comprehensive. Smoke free laws and } \\
\text { enforcement }\end{array}$ & $370(203,504)$ & $364(208,476)$ & $4783(2600,6589)$ & $12086(6695,16308)$ \\
\hline Comprehensive marketing ban & $298(163,408)$ & $326(185,429)$ & $3809(2063,5266)$ & $9966(5503,13506)$ \\
\hline High intensity tobacco control campaigns & $221(121,303)$ & $218(124,286)$ & $2778(1505,3840)$ & $7188(3966,9741)$ \\
\hline Strong health warnings & $307(167,424)$ & $344(195,452)$ & $3611(1941,5027)$ & $10205(5606,13885)$ \\
\hline Strong youth access enforcements & $8(4,12)$ & $110(53,173)$ & $18(9,27)$ & $1035(493,1618)$ \\
\hline Cessation treatment policies & $288(149,368)$ & $291(157,347)$ & $3200(1650,4190)$ & $9249(4843,11641)$ \\
\hline All above policies with $\$ 2$ increase in tax & $1853(1139,2314)$ & $2002(1326,2336)$ & $23206(14$ 158, 29 447) & $61626(38711,75$ 638) \\
\hline
\end{tabular}

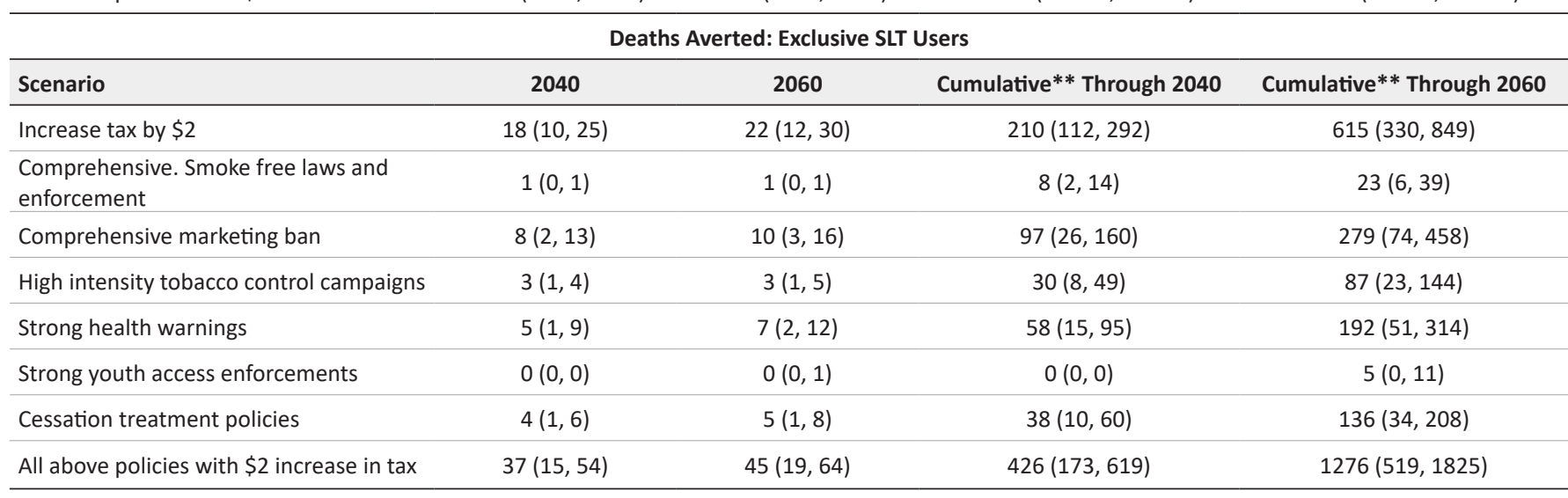

Abbreviation: SLT, smokeless tobacco.

* Lives saved is reported as the best estimate with its left/right bound range from model predictions using lower/upper policy effect sizes.

** Cumulative is reported from 2019 through a particular year.

overall smoking prevalence by $6.5 \%$ for both genders with 12086 fewer deaths, but with minimal effects on SLT use. A well-funded campaign reduces smoking prevalence by $3.5 \%$ with 7188 deaths averted but has limited SLT effects. Strict marketing restrictions reduce cigarette and SLT use by $7 \%$ with 9966 smoking and 279 SLT deaths averted. Strong health warnings reduce smoking by $6.5 \%$ with 10205 fewer smoking deaths and reduce SLT use by $4.7 \%$ with 192 fewer SLT-related deaths. Cessation policies reduce smoking prevalence by $3.2 \%$ with 9249 averted smoking deaths and reduce SLT use by 2.0\% with 136 averted SLT-related deaths. With strong youth policies, SimSmoke shows a reduction of $7 \%$ for cigarette and $1.5 \%$ for SLT use, averting 1035 smoking-related and 5 SLTrelated deaths by 2060 .

With the above policies in combination, SimSmoke predicts that by 2060 , male and female prevalence are reduced by $41 \%$, male and female exclusive SLT use are reduced by $33 \%$ and $25 \%$ respectively. Premature deaths are reduced by 61626 for smoking-attributable deaths and 1276 from exclusive SLTattributable deaths.

\section{Discussion}

Our projection of trends in cigarette and dual use from Kentucky SimSmoke are relatively well-supported by two large scale nationally representative surveys, TUS-CPS and BRFSS. However, the analysis of SLT does not fit the data as well. SimSmoke underestimated the reduction in SLT prevalence in sub-periods 1993-2002 and 2002-2007 in the TUS-CPS and then failed to detect the increase between 2007 and 2015 in the TUS-CPS, and the increase between 2011 and 2017 found from BRFSS.

Similar to previous literature, ${ }^{63,64}$ our analyses show that Kentucky cigarette and SLT rates decreased between 1993 and 2007, particularly for males. The greater observed than estimated SimSmoke reductions in SLT use through 2007 (48.5\% in TUS-CPS vs. $17.1 \%$ from SimSmoke) may reflect the indirect impact of cigarette-targeted tobacco control policies. The estimated effect sizes of cigarette-oriented policies on SLT use in SimSmoke reflects studies of use patterns prior to 2007. With major changes in tobacco control policies between 1993 and 2007, these findings suggest that cigarette-oriented policies may have additional impacts on SLT use. Since reductions in cigarette use were associated with reductions in SLT use, these patterns suggest a complementary relationship between the use of these two products. However, since dual use did not increase, these patterns may reflect changes in norms towards single tobacco use rather the dual use of the products.

Although not predicted by SimSmoke, SLT use increased in recent years according to the TUS-CPS and BRFSS estimates and other recent studies. ${ }^{65-70}$ These increases may reflect the growth in SLT marketing by cigarette manufacturers. ${ }^{71}$ The increase in unregulated sales of flavored SLT products also could contribute to smoking initiation at younger ages. ${ }^{72,73}$ 
Since 2006, cigarette companies Reynolds bought one of the largest SLT companies Conwood, and Altria bought US Smokeless Tobacco Company and both cigarette companies produced their own varieties of SLT (eg, Camel snus). ${ }^{74}$ During that period, cigarette companies increased the marketing of SLT, lowered prices and, in particular, marketed SLT as a product that could be used in places (eg, worksites) where smoking was not allowed. ${ }^{75,76}$ Similar and even stronger patterns of increased SLT use were observed in Minnesota, ${ }^{77}$ which implemented a statewide smoke-free air ban. The increase in unregulated sales of flavored SLT products also could have contributed to SLT initiation at younger ages, ${ }^{72,73}$ as indicated by the particularly large increase in exclusive and dual SLT use of young adults (especially ages 18-24). These patterns suggest that SLT use has acted as a substitute for cigarettes in recent years.

The findings in this study have implications for the recent growth in e-cigarette and heated tobacco product use. From 1993-2002, SLT use fell during a period of active cigarette-oriented policies. This finding suggests that more restrictive cigarette-oriented policies may also serve in a complementary fashion to reduce e-cigarette use. At the same time, e-cigarettes may be a better substitute for cigarettes than SLTs, since they appear to more efficiently deliver nicotine and provide sensorimotor effects closer to cigarettes than other non-combustible tobacco products. ${ }^{15}$ In that case, stronger policies may cause increased substitution of e-cigarettes for cigarettes, especially if e-cigarette policies are weak. In recent years (since 2006), cigarette and SLT companies have been more active in promoting new forms of oral tobacco products. ${ }^{10,11,78}$ The recent growth in SLT use by young adults also suggests that cigarette companies may actively promote e-cigarettes to be used with cigarettes (eg, dual use), again suggesting a complementary relationship. In particular, Altria recently purchased oral tobacco firm "On"10 and introduced heated tobacco products (ie, IQOS). With this increasing diversification of products, the landscape for nicotine delivery products is likely to become increasingly complex. ${ }^{79,80}$ It will be important to monitor the behavior of cigarette companies and how they promote products that may serve as an alternative to cigarettes.

While the current role of SLT use vis-à-vis e-cigarettes needs further policy consideration, there is a potential for further reductions in tobacco usage in Kentucky. For example, a SimSmoke model for Minnesota, ${ }^{62}$ one of the leading tobacco control policy states, estimates that policies implemented since 1993 led to a reduction in smoking prevalence of 35\% and SLT use by $23 \%$ by 2018 . By that same year, SimSmoke results for Kentucky estimate a smoking prevalence reduction of $24 \%$ and $5 \%$ for male SLT users. The variations are mostly due to the different levels of policies implemented. By 2018, Minnesota had a cigarette excise tax of \$3.04, smoke freeair laws in workplaces, schools, and restaurants and bars, an adequate level of cessation coverage, and spent $32 \%$ of the CDC's recommended budget for tobacco control. In contrast, Kentucky had an excise tax of $\$ 0.60$ and a third of the state with smoke-free air laws in schools, government buildings, workplaces, and restaurants, and the state spent $10 \%$ of the
CDC recommended level for tobacco control expenditures. ${ }^{5}$

Since early 2018, Kentucky has started to move towards more aggressive regulations by implementing a CDCrecommended comprehensive cessation program and increasing the cigarette tax in July 2018. An increase in the Kentucky cigarette excise tax of $\$ 0.50$ to $\$ 1.10^{81}$ could achieve a reduction in smoking prevalence of $2.8 \%$ in the first few years. If the cigarette tax is increased by an additional $\$ 0.90$ to $\$ 2.00$, Kentucky would then be above the mean US state taxation of $\$ 1.82$, which would result in further reducing smoking prevalence by $5.1 \%$ by 2060 .

Like all models, our results are only as strong as the assumptions and underlying data. We assumed that projections of cigarette use are based on initiation and cessation rates from 1993, but subject to policy changes over time. Thus, the initiation and cessation rate estimated using smoking prevalence data from 1993, and the policy levels and effect sizes play an important role. Also, we do not explicitly incorporate the role of industry, which may have had a major impact when the major SLT producing firms were acquired by cigarette manufacturers. ${ }^{74}$ In addition, cessation and relapse data were not available for SLT use. Also, we treated SLT as a homogeneous category in terms of risks and an ability to substitute for cigarettes, although new forms, such as snus and other oral tobacco products, have come onto the market. ${ }^{10,11}$

Another limitation of the model is that includes only cigarettes and SLT use and does not incorporate the use of other nicotine delivery products, including cigars, water pipes, heated tobacco products, and e-cigarettes. Each may act as substitute or complement to the use of cigarettes and SLT. While use of these products may be a relatively minor contributor to overall tobacco-related harms, policies should be directed at all of these products, particularly small cigars. ${ }^{82}$

Finally, we assume that cigarette-oriented and SLT-oriented policies have independent effects. As suggested above, cigarette-oriented policies may have indirect impacts on SLT use through social norms. The literature on the interrelationship between the effects of different types of policies is sparse. ${ }^{42,62}$ While some smokeless demand studies incorporate cigarette prices and some cigarette demand studies incorporate smokeless prices, ${ }^{40-42,64}$ the studies obtain mixed results regarding whether the two products are substitutes or complements. ${ }^{42}$ In general, greater information is needed on the inter-related effects of policies targeting different tobacco products.

\section{Conclusion}

While the landscape for nicotine delivery products has dramatically changed in the last 10 years, some lessons can be gleaned from our results. First, with cigarettes still the dominant form of nicotine delivery, cigarette-oriented policies appear to be an effective means of reducing the use of nicotine delivery products. However, with SLT use increasing in recent years, policies directed at SLT use may also play a role. With cigarette manufacturers having acquired major SLT firms, it is important to monitor the role of the cigarette industry. With strong incentives to protect the high profits from cigarettes, cigarette firms can be expected to encourage 
dual use rather than switching to other tobacco products. Well-targeted policies and regulations, such as cigarette and SLT tax increases and media campaigns, will be needed to achieve reductions in SLT use.

\section{Ethical issues}

None, the study uses publicly available data.

\section{Competing interests}

Authors declare that they have no competing interests.

\section{Authors' contributions}

LMSR wrote and edited much of the original draft. DTL conceived the idea and edited and revised the paper, $Z Y$ and $Y L$ conducted the modeling, wrote up results and reviewed the manuscript.

\section{Disclaimer}

The content is solely the responsibility of the authors and does not necessarily represent the official views of the National Institute on Drug Abuse of the National Institutes of Health.

\section{Funding}

This work was supported by the National Institute on Drug Abuse of the National Institutes of Health under grant R01DA036497.

\section{References}

1. Centers for Disease Control and Prevention (CDC). BRFSS Prevalence \& Trends Data. CDC; 2020. https://nccd.cdc.gov/ BRFSSPrevalence/rdPage.aspx?rdReport=DPH_BRFSS.ExploreBy Topic\&irbLocationType=StatesAndMMSA\&isIClass=CLASS17\&isITo pic=TOPIC15\&islYear=2018\&rdRnd=91004. Accessed July 3, 2020.

2. Centers for Disease Control and Prevention (CDC). National Health Interview Survey. CDC; 2019.

3. Levy DT, Tworek C, Hahn EJ, Davis RE. The Kentucky SimSmoke tobacco policy simulation model: reaching Healthy People 2010 goals through policy change. South Med J. 2008;101(5):503-507. doi:10.1097/SMJ.0b013e31816c013c

4. American Lung Association. 'State of Tobacco Control' Report: Kentucky Gets Mostly Failing Grades for Work to Prevent Tobacco Use, Prioritize Public Health. Louisville, Kentucky: American Lung Association; 2020. https://www.lung.org/media/press-releases/stateof-tobacco-control-9. Accessed July 28, 2020.

5. Campaign for Tobacco-Free Kids. Broken Promises to Our Children: A State-by-State Look at the 1998 Tobacco Settlement 20 Years Later. Washington, DC: Campaign for Tobacco-Free Kids; 2018.

6. Nguyen K, Marshall L, Hu S, Neff L. State-specific prevalence of current cigarette smoking and smokeless tobacco use among adults aged $\geq 18$ years - United States, 2011-2013. MMWR Morb Mortal Wkly Rep. 2015;64(19):532-536.

7. Nguyen KH, Marshall L, Brown S, Neff L. State-specific prevalence of current cigarette smoking and smokeless tobacco use among adults - United States, 2014. MMWR Morb Mortal Wkly Rep. 2016; 65(39):1045-1051. doi:10.15585/mmwr.mm6539a1

8. Hu SS, Homa DM, Wang T, et al. State-specific patterns of cigarette smoking, smokeless tobacco use, and e-cigarette use among adults - United States, 2016. Prev Chronic Dis. 2019;16:E17. doi:10.5888/ pcd16.180362

9. High School Youth Risk Behavior Survey. Kentucky 2017 and United States 2017 Results. Centers for Disease Control and Prevention; 2017.

10. Altria Enters Growing Oral Nicotine Products Category with on! Pouch Product. Bloomberg. June 3, 2019. https://www.bloomberg. com/press-releases/2019-06-03/altria-enters-growing-oral-nicotineproducts-category-with-on-pouch-product.

11. Plurphanswat N, Hughes JR, Fagerström K, Rodu B. Initial information on a novel nicotine product. Am J Addict. 2020;29(4):279-286. doi:10.1111/ajad.13020

12. Levy DT, Mumford EA, Cummings KM, et al. The relative risks of a low-nitrosamine smokeless tobacco product compared with smoking cigarettes: estimates of a panel of experts. Cancer Epidemiol Biomarkers Prev. 2004;13(12):2035-2042.
13. Henley SJ, Thun MJ, Connell C, Calle EE. Two large prospective studies of mortality among men who use snuff or chewing tobacco (United States). Cancer Causes Control. 2005;16(4):347-358. doi:10.1007/s10552-004-5519-6

14. Sinha DN, Suliankatchi RA, Gupta PC, et al. Global burden of allcause and cause-specific mortality due to smokeless tobacco use: systematic review and meta-analysis. Tob Control. 2018;27(1):35-42. doi:10.1136/tobaccocontrol-2016-053302

15. Levy DT, Cummings KM, Villanti AC, et al. A framework for evaluating the public health impact of e-cigarettes and other vaporized nicotine products. Addiction. 2017;112(1):8-17. doi:10.1111/add.13394

16. Levy DT, Yuan Z, Li Y. The US SimSmoke tobacco control policy model of smokeless tobacco and cigarette use. BMC Public Health. 2018;18(1):696. doi:10.1186/s12889-018-5597-0

17. Levy D, Sanchez-Romero L, Yuan Z, Li Y. The Kentucky SimSmoke Tobacco Policy Simulation Model of Cigarette and Smokeless Tobacco Use. Washington, DC: Georgetown University; 2019.

18. State and County Intercensal Datasets: 1990-2000. United States Census Bureau; 2017. https://www.census.gov/data/datasets/ time-series/demo/popest/intercensal-1990-2000-state-and-countycharacteristics.html. Accessed May 2019.

19. Population projections. http://www.ksdc.louisville.edu/data-downloads/projections/. Accessed June 20, 2017. Published 2016.

20. Fertility rates, mortality and birth rates by age and gender. http:// wonder.cdc.gov/cmf-icd10.html. Published 2015. Accessed August 15, 2015.

21. United States Census Bureau. Current Population Survey, September 1993: Tobacco Use Supplement File, Technical Documentation CPS01. Washington, DC: United States Census Bureau; 2001.

22. Hatsukami DK, Henningfield JE, Kotlyar M. Harm reduction approaches to reducing tobacco-related mortality. Annu Rev Public Health. 2004;25:377-395. doi:10.1146/annurev.publhealth.25.102802.124406

23. Hatsukami DK, Lemmonds C, Tomar SL. Smokeless tobacco use: harm reduction or induction approach? Prev Med. 2004;38(3):309317. doi:10.1016/j.ypmed.2003.10.006

24. Phillips CV, Heavner KK. Smokeless tobacco: the epidemiology and politics of harm. Biomarkers. 2009;14 Suppl 1:79-84. doi:10.1080/13547500902965476

25. Tomar SL. Is use of smokeless tobacco a risk factor for cigarette smoking? the U.S. experience. Nicotine Tob Res. 2003;5(4):561-569. doi:10.1080/1462220031000118667

26. Tomar SL. Epidemiologic perspectives on smokeless tobacco marketing and population harm. Am J Prev Med. 2007;33(6 Suppl): S387-397. doi:10.1016/j.amepre.2007.09.009

27. Zhu SH, Wang JB, Hartman A, et al. Quitting cigarettes completely or switching to smokeless tobacco: do US data replicate the Swedish results? Tob Control. 2009;18(2):82-87. doi:10.1136/tc.2008.028209

28. Tam J, Day HR, Rostron BL, Apelberg BJ. A systematic review of transitions between cigarette and smokeless tobacco product use in the United States. BMC Public Health. 2015;15:258. doi:10.1186/ s12889-015-1594-8

29. Chang JT, Levy DT, Meza R. Examining the transitions between cigarette and smokeless tobacco product use in the United States using the 2002-2003 and 2010-2011 longitudinal cohorts. Nicotine Tob Res. 2018;20(11):1412-1416. doi:10.1093/ntr/ntx251

30. Burns DM, Anderson C, Major J, Vaughn J, Shanks T. Cessation and cessation measures among daily adult smokers: National- and Statespecific data. Population Based Smoking Cessation Monograph No 12. Washington, DC: National Institutes of Health. National Cancer Institute; 2000.

31. McWhorter WP, Boyd GM, Mattson ME. Predictors of quitting smoking: the NHANES I followup experience. J Clin Epidemiol. 1990; 43(12):1399-1405. doi:10.1016/0895-4356(90)90108-2

32. Gilpin EA, Pierce JP, Farkas AJ. Duration of smoking abstinence and success in quitting. J Natl Cancer Inst. 1997;89(8):572-576. doi:10.1093/jnci/89.8.572

33. United States Department of Health and Human Services. Reducing the Health Consequences of Smoking: 25 Years of Progress: A Report of the Surgeon General. Atlanta, GA: Centers for Disease Control and Prevention, National Center for Chronic Disease Prevention and Health Promotion, Office on Smoking and Health; 1989.

34. United States Department of Health and Human Services. The Health 
Benefits of Smoking Cessation: A Report of the Surgeon General. Atlanta, GA: United States Department of Health and Human Services, Public Health Service, Centers for Disease Control, Office on Smoking and Health; 1990.

35. Schauer GL, Pederson LL, Malarcher AM. Past year quit attempts and use of cessation resources among cigarette-only smokers and cigarette smokers who use other tobacco products. Nicotine Tob Res. 2016;18(1):41-47. doi:10.1093/ntr/ntv038

36. Messer K, Vijayaraghavan M, White MM, et al. Cigarette smoking cessation attempts among current US smokers who also use smokeless tobacco. Addict Behav. 2015;51:113-119. doi:10.1016/j. addbeh.2015.06.045

37. Thun MJ, Myers DG, Day-Lally C, et al. Age and the exposureresponse relationships between cigarette smoking and premature death in Cancer Prevention Study II. In: Changes in Cigarette-Related Disease Risks and Their Implications for Prevention and Control. Bethesda, MD: National Cancer Institute; 1997:383-475.

38. Burns D, Garfinkel L, Samet J, eds. Changes in Cigarette-Related Disease Risks and Their Implications for Prevention and Control. Bethesda, MD: National Institutes of Health, National Cancer Institute; 1997.

39. Levy DT, Cummings KM, Hyland A. Increasing taxes as a strategy to reduce cigarette use and deaths: results of a simulation model. Prev Med. 2000;31(3):279-286. doi:10.1006/pmed.2000.0696

40. Huang J, Gwarnicki C, Xu X, Caraballo RS, Wada R, Chaloupka FJ. A comprehensive examination of own- and cross-price elasticities of tobacco and nicotine replacement products in the U.S. Prev Med. 2018:117:107-114. doi:10.1016/j.ypmed.2018.04.024

41. Zheng Y, Zhen C, Dench D, Nonnemaker JM. U.S. demand for tobacco products in a system framework. Health Econ. 2017;26(8):1067-1086. doi:10.1002/hec.3384

42. Levy DT, Mays D, Boyle RG, Tam J, Chaloupka FJ. The effect of tobacco control policies on US smokeless tobacco use: a structured review. Nicotine Tob Res. 2017;20(1):3-11. doi:10.1093/ntr/ntw291

43. Orzechowski and Walker. TThe Tax Burden on Tobacco Volume 52, 1970-2018. Arlington, VA: Orzechowski and Walker; 2018.

44. Federal Trade Commission Smokeless Tobacco Report for 2013: Federal Trade Commission. USA; 2016. https://www.ftc.gov/system/ files/documents/reports/federal-trade-commission-smokelesstobacco-report-2013/2013tobaccorpt.pdf. Accessed 2019.

45. U.S. Bureau of Labor Statistics. Databases, Tables \& Calculators by Subject: United States Department of Labor. https://data.bls.gov/ timeseries/CUUR0000SA0. Accessed 2019.

46. Levy DT, Friend K, Polishchuk E. Effect of clean indoor air laws on smokers: the clean air module of the SimSmoke computer simulation model. Tob Control. 2001;10(4):345-351. doi:10.1136/tc.10.4.345

47. Chronological Table of U.S. Population Protected by $100 \%$ Smokefree State or Local Laws. http://no-smoke.org/pdf/EffectivePopulationList. pdf. Accessed February 8, 2019

48. Huang J, Walton K, Gerzoff RB, King BA, Chaloupka FJ. State tobacco control program spending--United States, 2011. MMWR Morb Mortal Wkly Rep. 2015;64(24):673-678.

49. Levy DT, Friend K. A computer simulation model of mass media interventions directed at tobacco use. Prev Med. 2001;32(3):284-294. doi:10.1006/pmed.2000.0808

50. Factsheet: State Tobacco Control Expenditures, 2000-2016. http://www.tobaccofreekids.org/research/factsheets/pdf/0209.pdf. Accessed September 2, 2017. Published 2017.

51. Levy DT, Mays D, Yuan Z, Hammond D, Thrasher JF. Public health benefits from pictorial health warnings on US cigarette packs: a SimSmoke simulation. Tob Control. 2017;26(6):649-655. doi:10.1136/ tobaccocontrol-2016-053087

52. Wagener TL, Meier E, Hale JJ, et al. Pilot investigation of changes in readiness and confidence to quit smoking after E-cigarette experimentation and 1 week of use. Nicotine Tob Res. 2014;16(1):108114. doi:10.1093/ntr/ntt138

53. Levy DT, Graham AL, Mabry PL, Abrams DB, Orleans CT. Modeling the impact of smoking-cessation treatment policies on quit rates. Am J Prev Med. 2010;38(3 Suppl):S364-372. doi:10.1016/j. amepre.2009.11.016

54. Quitline Stats. http://www.naquitline.org/?page $=800 \mathrm{QUITNOW}$ stats. Accessed August 30, 2015. Published 2015.
55. van Zyl-Smit RN. Electronic cigarettes: the potential risks outweigh the benefits. S Afr Med J. 2013;103(11):833.

56. CHFS. Tobacco Use in Kentucky 2012. Frankfort, KY, US: Kentucky Department for Public Health; 2012.

57. Levy DT, Friend K, Holder H, Carmona M. Effect of policies directed at youth access to smoking: results from the SimSmoke computer simulation model. Tob Control. 2001;10(2):108-116. doi:10.1136/ tc. 10.2 .108

58. Clark PI, Natanblut SL, Schmitt CL, Wolters C, lachan R. Factors associated with tobacco sales to minors: lessons learned from the FDA compliance checks. JAMA. 2000;284(6):729-734. doi:10.1001/ jama.284.6.729

59. Choi K, Fabian LE, Brock B, Engman KH, Jansen J, Forster $\mathrm{JL}$. Availability of snus and its sale to minors in a large Minnesota city. Tob Control. 2014;23(5):449-451. doi:10.1136/ tobaccocontrol-2012-050719

60. Chaloupka FJ, Tauras JA, Grossman M. Public policy and youth smokeless tobacco use. South Econ J. 1997;64:503-516.

61. Tauras J, Powell L, Chaloupka F, Ross H. The demand for smokeless tobacco among male high school students in the United States: the impact of taxes, prices and policies. Appl Econ. 2007;39(1):31-41. doi:10.1080/00036840500427940

62. Levy DT, Tam J, Kuo C, Fong GT, Chaloupka F. The impact of implementing tobacco control policies: the 2017 tobacco control policy scorecard. J Public Health Manag Pract. 2018;24(5):448-457. doi:10.1097/phh.0000000000000780

63. Nelson DE, Mowery P, Tomar S, Marcus S, Giovino G, Zhao L. Trends in smokeless tobacco use among adults and adolescents in the United States. Am J Public Health. 2006;96(5):897-905. doi:10.2105/ ajph.2004.061580

64. Mumford EA, Levy DT, Gitchell JG, Blackman KO. Tobacco control policies and the concurrent use of smokeless tobacco and cigarettes among men, 1992-2002. Nicotine Tob Res. 2005;7(6):891-900. doi:10.1080/14622200500266098

65. Agaku IT, Alpert HR. Trends in annual sales and current use of cigarettes, cigars, roll-your-own tobacco, pipes, and smokeless tobacco among US adults, 2002-2012. Tob Control. 2016;25(4):451457. doi:10.1136/tobaccocontrol-2014-052125

66. Bhattacharyya N. Trends in the use of smokeless tobacco in United States, 2000-2010. Laryngoscope. 2012;122(10):2175-2178. doi:10.1002/lary. 23448

67. Chang JT, Levy DT, Meza R. Trends and factors related to smokeless tobacco use in the United States. Nicotine Tob Res. 2016;18(8):17401748. doi:10.1093/ntr/ntw090

68. Giovenco DP, Hammond D, Corey CG, Ambrose BK, Delnevo CD. E-cigarette market trends in traditional U.S. retail channels, 2012 2013. Nicotine Tob Res. 2015;17(10):1279-1283. doi:10.1093/ntr/ ntu282

69. U.S. Department of Health and Human Services. The Health Consequences of Smoking-50 Years of Progress: A Report of the Surgeon General. Atlanta, GA: U.S. Department of Health and Human Services; 2014.

70. Delnevo CD, Wackowski OA, Giovenco DP, Manderski MT, Hrywna M, Ling PM. Examining market trends in the United States smokeless tobacco use: 2005-2011. Tob Control. 2014;23(2):107-112. doi:10.1136/tobaccocontrol-2012-050739

71. Economic Trends in Tobaco. Tobacco-Related Spending. CDC; 2018. https://www.cdc.gov/tobacco/data_statistics/fact_sheets/economics/ econ_facts/index.htm. Accessed May 31, 2019.

72. Kostygina G, Ling PM. Tobacco industry use of flavourings to promote smokeless tobacco products. Tob Control. 2016;25(Suppl 2):ii40-ii49. doi:10.1136/tobaccocontrol-2016-053212

73. Morris DS, Fiala SC. Flavoured, non-cigarette tobacco for sale in the USA: an inventory analysis of Internet retailers. Tob Control. 2015;24(1):101-102. doi:10.1136/tobaccocontrol-2013-051059

74. National Cancer Institute $(\mathrm{NCl})$, Centers for Disease Control and Prevention (CDC). Smokeless Tobacco and Public Health: A Global Perspective. Washington, DC: NCl, CDC; 2014.

75. Mejia AB, Ling PM. Tobacco industry consumer research on smokeless tobacco users and product development. Am J Public Health. 2010;100(1):78-87. doi:10.2105/ajph.2008.152603

76. Carpenter CM, Connolly GN, Ayo-Yusuf OA, Wayne GF. Developing 
smokeless tobacco products for smokers: an examination of tobacco industry documents. Tob Control. 2009;18(1):54-59. doi:10.1136/ tc. 2008.026583

77. Levy DT, Yuan Z, Li Y, St Claire AW, Schillo BA. The Minnesota SimSmoke tobacco control policy model of smokeless tobacco and cigarette use. Am J Prev Med. 2019;57(4):e103-e115. doi:10.1016/j. amepre.2019.06.003

78. Lunell E, Fagerström K, Hughes J, Pendrill R. Pharmacokinetic comparison of a novel non-tobacco-based nicotine pouch (ZYN®) with conventional, tobacco-based Swedish snus and American moist snuff. Nicotine Tob Res. 2020. doi:10.1093/ntr/ntaa068

79. Max WB, Sung HY, Lightwood J, Wang Y, Yao T. Modelling the impact of a new tobacco product: review of Philip Morris International's
Population Health Impact Model as applied to the IQOS heated tobacco product. Tob Control. 2018;27(Suppl 1):s82-s86. doi:10.1136/ tobaccocontrol-2018-054572

80. McKelvey K, Baiocchi M, Halpern-Felsher B. PMI's heated tobacco products marketing claims of reduced risk and reduced exposure may entice youth to try and continue using these products. Tob Control. 2020. doi:10.1136/tobaccocontrol-2019-055318

81. Key State-Specific Tobacco-Related Data \& Ranking. Tobacco free kids website. https://www.tobaccofreekids.org/assets/factsheets/0176.pdf. Accessed May 31, 2019. Published 2019.

82. Delnevo CD, Hrywna M, Foulds J, Steinberg MB. Cigar use before and after a cigarette excise tax increase in New Jersey. Addict Behav. 2004;29(9):1799-1807. doi:10.1016/j.addbeh.2004.04.024 